\title{
Synthesis of heptasaccharide and nonasaccharide analogues of the lentinan repeating unit
}

\author{
Guangbin Yang and Fanzuo Kong* \\ Research Center for Eco-Environmental Sciences, Academia Sinica, PO Box 2871, Beijing 100085, China
}

Received 25 August 2004; accepted 3 November 2004

Available online 26 November 2004

\begin{abstract}
The allyl glycoside $\quad \beta$-D-Glc $p$ - $(1 \rightarrow 3)-\beta$-D-Glc $p-(1 \rightarrow 3)-[\beta-\mathrm{D}-\mathrm{Glc} p-(1 \rightarrow 6)]-\beta-\mathrm{D}-\mathrm{Glc} p-(1 \rightarrow 3)-\beta$-D-Glc $p-(1 \rightarrow 3)-[\beta-\mathrm{D}-\mathrm{Glc} p-$ $(1 \rightarrow 6)]-\alpha-D-G l c p(\mathbf{1 8})$ and the acetonyl glycoside of $\beta$-D-Glc $p-(1 \rightarrow 3)-[\beta-D-G l c p-(1 \rightarrow 6)]-\beta-D-G l c p-(1 \rightarrow 3)-\beta-D-G l c p-(1 \rightarrow 3)-[\beta-D-$ Glcp-( $1 \rightarrow 6)]-\beta-\mathrm{D}-\mathrm{Glc} p-(1 \rightarrow 3)-\beta-\mathrm{D}-\mathrm{Glc} p-(1 \rightarrow 3)-[\beta-\mathrm{D}-\mathrm{Glc} p-(1 \rightarrow 6)]-\alpha-\mathrm{D}-\mathrm{Glc} p(\mathbf{2 8})$ were synthesized as analogues of the lentinan heptaose repeating unit. 4,6- $O$-Benzylidenated monosaccharide donor 3 and 4,6- $O$-benzylidenated tetrasaccharide acceptor 14 were used to ensure the $\beta$-linkage in the synthesis of $\mathbf{1 8}$, while 4,6-O-benzylidenated disaccharide acceptor 20, and 4,6- $O$-benzylidenated disaccharide donors $\mathbf{2 1}$ and $\mathbf{2 4}$ were used to ensure the $\beta$-linkage in the synthesis of $\mathbf{2 8}$.
\end{abstract}

(C) 2004 Elsevier Ltd. All rights reserved.

Keywords: Trichloroacetimidate; $\beta-(1 \rightarrow 6)$-Branched $\beta-(1 \rightarrow 3)$-glucan; Synthesis

\section{Introduction}

Polysaccharides with antitumor activity separated from fungi such as Ganoderma lucidum, Schizophyllum commune, and Lentinus edodes have a $\beta-(1 \rightarrow 3)$-linked glucosyl backbone with $\beta-(1 \rightarrow 6)$-branched glucosyl side chains. ${ }^{1}$ Some physicochemical and immunopharmacological investigations showed that the antitumor activity of these glucans may be related to the triple-helix structures of the $\beta-(1 \rightarrow 3)$-linked backbone chains, ${ }^{2 a, b}$ and some biological aspects of $\beta$-glucans have been reported. ${ }^{2 \mathrm{c}-\mathrm{e}}$ It was also reported that only higher molecular-weight fractions (MW >16,000) obtained from partial hydrolysis of lentinan with formic acid showed antitumor activity. ${ }^{3}$ However, an interesting result in our research revealed that a synthetic allyl glycoside of $\beta$-D-Glc $p-(1 \rightarrow 3)-[\beta-\mathrm{D}-\mathrm{Glc} p-(1 \rightarrow 6)]-\beta-\mathrm{D}-\mathrm{Gl} p-(1 \rightarrow 3)-$ $\beta$-D-Glc $p$ - $(1 \rightarrow 3)-[\beta-\mathrm{D}-\mathrm{Glc} p-(1 \rightarrow 6)]-\beta-\mathrm{D}-\mathrm{Glc} p-(1 \rightarrow 3)-\alpha-\mathrm{D}-$ Glc $p^{4}$ at a dose of $5 \mathrm{mg} / \mathrm{kg}$ effectively inhibited the $\mathrm{U}_{14}$ tumor $(58.4 \%){ }^{5}$ Encouraged by the bioassay results, we are trying to synthesize more structurally diverse 3,6-branched glucans and investigate structure-activity relationships. The major structure of lentinan consists of a glucoheptaose repeating unit as shown in Figure 1 , and its synthesis was reported by our group. ${ }^{4}$

Heptaoside analogues with the $\beta$ - $(1 \rightarrow 3)$-linked pentaose backbone and C-6, C- 6 "' branches, and C- 6 disaccharide branch were also synthesized. ${ }^{6} \mathrm{We}$ present

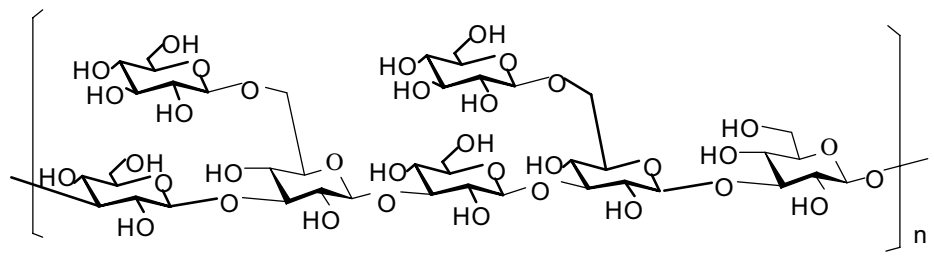

Figure 1. Structure of lentinan.

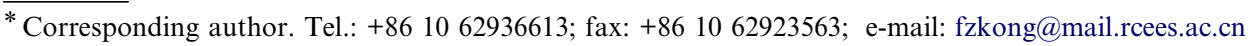


herein the syntheses of allyl glucoheptaoside 18 consisting of the $\beta-(1 \rightarrow 3)$-linked pentaose backbone with $\beta$ $(1 \rightarrow 6)$-linked glucosyl side chains attached at C-6 and C- 6 " of the backbone, and acetonyl glucononaoside $\mathbf{2 8}$ consisting of a $\beta-(1 \rightarrow 3)$-linked hexaose backbone with glucosyl side chains linked at C-6, C-6", and C-6"'", respectively.

\section{Results and discussion}

Scheme 1 shows the synthesis of the glucoheptaoside 18. Allyl 3-O-chloroacetyl-2- $O$-benzoyl-4,6- $O$-benzylidene- $\alpha$-D-glucopyranoside ${ }^{4}(\mathbf{1})$, allyl 4,6-di- $O$-acetyl-3$O$-chloroacetyl-2- $O$-benzoyl- $\beta$-D-glucopyranosyl- $(1 \rightarrow 3)$ 2-O-benzoyl-4,6- $O$-benzylidene- $\alpha$-D-glucopyranoside ${ }^{4}(4)$, and 2,3,4,6-tetra- $O$-benzoyl- $\alpha$-D-glucopyranosyltrichloroacetimidate $^{7}$ (9) were used as the key synthons. Deallylation of 1 with $\mathrm{PdCl}_{2}$ in acetic acid containing sodium acetate gave $\mathbf{2}$, and the benzylidene group was not affected under the mild conditions. Subsequent trichloroacetimidate formation with trichloroacetonitrile in the presence of DBU afforded the glycosyl donor 3 . Meanwhile, removal of the benzylidene group of $\mathbf{4}$ in glycol-acetonitrile with toluenesulfonic acid furnished the diol acceptor 5, while subsequent acetylation of $\mathbf{5}$, deallylation, and trichloroacetimidate formation produced the disaccharide donor 8. Condensation of the diol 5 with 9 selectively yielded $(1 \rightarrow 6)$-linked trisaccharide 10, then acetylation and dechloroacetylation with thiourea gave the trisaccharide acceptor 12. Consequently, coupling of $\mathbf{1 2}$ with the donor $\mathbf{3}$, followed by dechloroacetylation, furnished the tetrasaccharide acceptor 14, and subsequent coupling of 14 with the disaccharide donor $\mathbf{8}$ gave the hexasaccharide $\mathbf{1 5}$. Debenzylidenation of $\mathbf{1 5}$ followed by selective coupling with 9 afforded the heptasaccharide 17. Finally, deprotection produced the target heptaoside $\mathbf{1 8}$.

Scheme 2 shows the synthesis of glucononaoside 28. First, acetonyl 4,6-di- $O$-acetyl-3- $O$-chloroacetyl-2- $O$ benzoyl- $\beta$-D-glucopyranosyl- $(1 \rightarrow 3)-2-O$-benzoyl-4,6- $O$ benzylidene- $\alpha$-D-glucopyranoside (19) was obtained by oxidation of the corresponding allyl glycoside of disaccharide 4 with $\mathrm{PdCl}_{2}$. Actually, our original goal was to remove the allyl group of $\mathbf{4}$ to obtain the corresponding hemiacetal. However, it was found that treatment of 4 in tetrahydrofuran, which was not purged with nitrogen to remove the air, with $\mathrm{PdCl}_{2}$ gave 19 and the hemiacetal in almost equal amount, and the two products were easily separated. Similar oxidation of the allyl group to the acetonyl group was reported many years ago. ${ }^{8}$ The disaccharide acceptor $\mathbf{2 0}$ was readily obtained by dechloroacetylation of $\mathbf{1 9}$ with thiourea. Condensation of 20 with 4,6-di- $O$-acetyl-3- $O$-chloroacetyl-2- $O$-benzoyl- $\beta$-D-glucopyranosyl-( $1 \rightarrow 3)-2-O$-benzoyl-4,6- $O$-benz- ylidene- $\alpha$-D-glucopyranosyl trichloroacetimidate ${ }^{4}$ (21), followed by dechloroacetylation, afforded the tetrasaccharide acceptor 23. Subsequent coupling of $\mathbf{2 3}$ with the disaccharide donor $24^{6}$ produced the hexasaccharide 25 with benzylidene groups at the 4,6-, 4", $6^{\prime \prime}$-, and $4^{\prime \prime \prime \prime}, 6^{\prime \prime \prime \prime}$-positions, respectively. Removal of the benzylidene groups of $\mathbf{2 5}$, followed by selective coupling with $\mathbf{9}$, and then deacylation, gave the target nonaoside $\mathbf{2 8}$.

In summary, with benzylidenated monosaccharide and disaccharide donors, and benzylidenated oligosaccharide acceptors, $\beta-(1 \rightarrow 3)$-linked glucans were readily obtained, and the formation ${ }^{9}$ of $\alpha$-linkages was restrained.

Preliminary bioassays for $\mathbf{1 8}$ and $\mathbf{2 8}$ revealed that $\mathbf{1 8}$ had activity similar to the lentinan heptaoside, ${ }^{4,5}$ while $\mathbf{2 8}$ showed even better activity.

\section{Experimental}

\subsection{General methods}

Melting points were determined with a 'Mel-Temp' apparatus. Optical rotations were determined with a Perkin-Elmer model 241-MC automatic polarimeter for solutions in a $1 \mathrm{dm}$, jacketed cell. ${ }^{1} \mathrm{H}$ NMR and ${ }^{13} \mathrm{C}$ NMR spectra were recorded with Varian XL-400 spectrometers, for solutions in $\mathrm{CDCl}_{3}$ or in $\mathrm{D}_{2} \mathrm{O}$ as indicated, and individual resonances could not be identified with the specific sugar residues using 1D techniques. Chemical shifts are expressed in ppm downfield from the $\mathrm{Me}_{4} \mathrm{Si}$ absorption. Mass spectra were recorded with a VG PLATFORM mass spectrometer operating in the ESI mode. Thin-layer chromatography (TLC) was performed on silica gel HF with detection by charring with $30 \%(\mathrm{v} / \mathrm{v})$ sulfuric acid in methanol or by UV detection. Column chromatography was conducted by elution of a column $(8 \times 100 \mathrm{~mm}, 16 \times 240 \mathrm{~mm}, \quad 18 \times 300 \mathrm{~mm}$, $35 \times 400 \mathrm{~mm})$ of silica gel (100-200 mesh) with EtOAcpetroleum ether (bp $60-90^{\circ} \mathrm{C}$ ) as the eluent. Analytical LC was performed with a Gilson HPLC consisting of a pump (model 306), stainless steel column packed with silica gel (Spherisorb $\mathrm{SiO}_{2}, 10 \times 300 \mathrm{~mm}$ or $4.6 \times 250 \mathrm{~mm})$, differential refractometer (132-RI Detector), UV/vis detector (model 118). EtOAc-petroleum ether (bp $60-90^{\circ} \mathrm{C}$ ) was used as the eluent at a flow rate of $1-4 \mathrm{~mL} / \mathrm{min}$. Solutions were concentrated at a temperature $<60^{\circ} \mathrm{C}$ under diminished pressure.

\subsection{General procedure for trichloroacetimidate formation}

To a solution of the hemiacetal $(0.4-2.2 \mathrm{mmol})$ in dry $\mathrm{CH}_{2} \mathrm{Cl}_{2}(10-25 \mathrm{~mL})$ were added $\mathrm{CCl}_{3} \mathrm{CN}$ (3 equiv) and DBU $(10-55 \mu \mathrm{L})$. The mixture was stirred at $\mathrm{rt}$ for $2 \mathrm{~h}$, at the end of which time TLC (3:1 petroleum ether- 

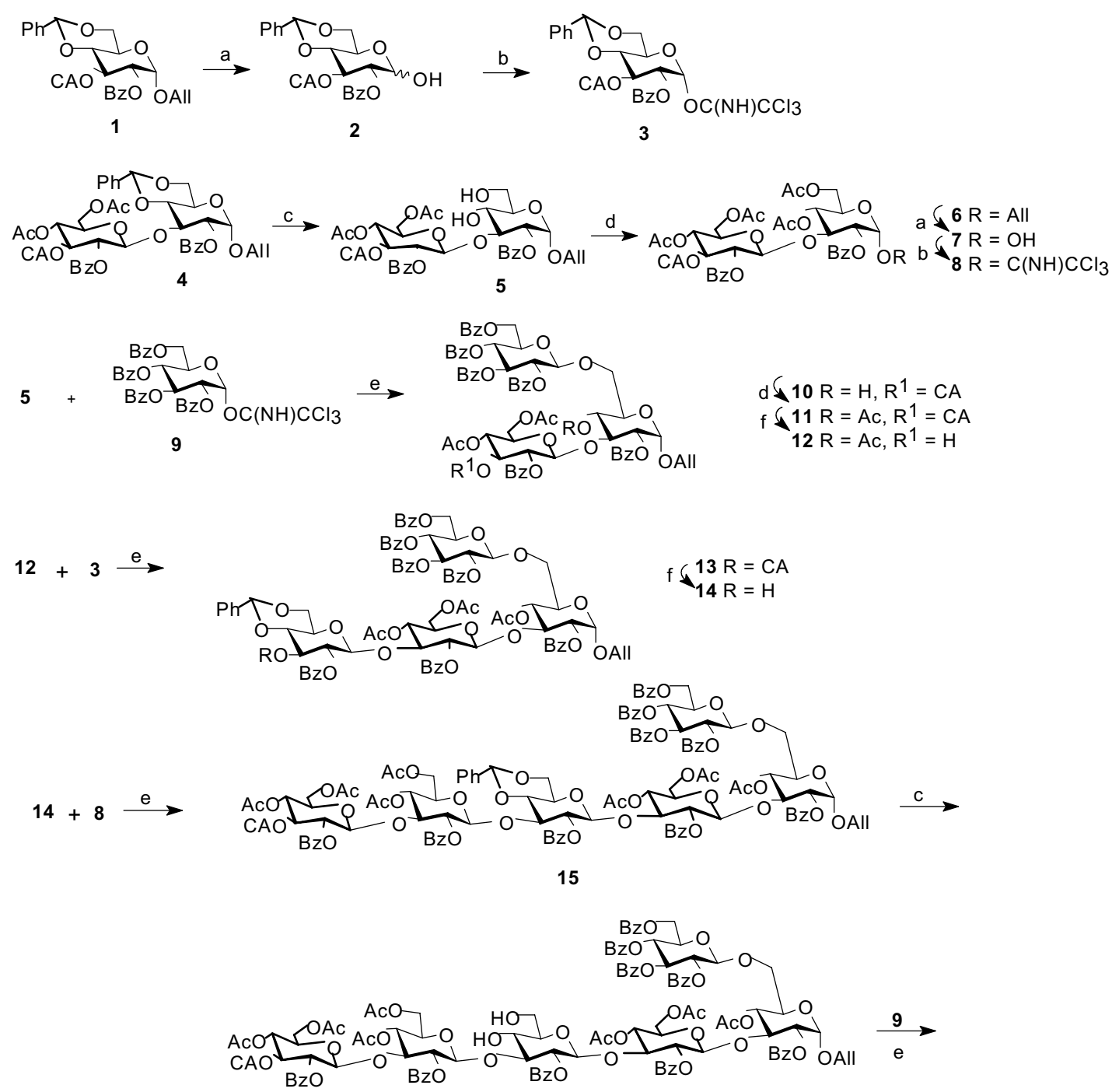

16
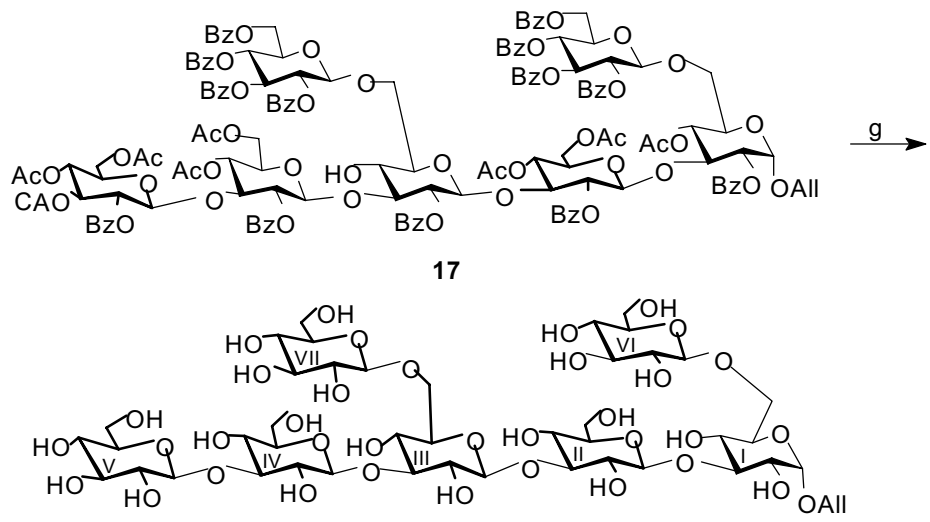

18

Scheme 1. Reagents and conditions: (a) $\mathrm{PdCl}_{2}, 75 \% \mathrm{HOAc}(75 \%)-\mathrm{NaOAc}$ (or $\mathrm{MeOH}$ ), rt, $80 \%$ for $\mathbf{2}, 82 \%$ for 7; (b) $\mathrm{DBU}, \mathrm{Cl}_{3} \mathrm{CCN}, \mathrm{CH}_{2} \mathrm{Cl}_{2}, \mathrm{rt}, 82 \%$ for 3, $87 \%$ for 8; (c) $\mathrm{C}_{2} \mathrm{H}_{4}(\mathrm{OH})_{2}, \mathrm{MeCN}$, TsOH, rt, $91 \%$ for 5, 79\% for 16; (d) $\mathrm{Ac}_{2} \mathrm{O}, \mathrm{Pyr}$, (or $\mathrm{AcCl}, \mathrm{Pyr}, \mathrm{CH}_{2} \mathrm{Cl}_{2}$ ), rt, $98 \%$ for 6, 90\% for 11; (e) TMSOTf, $\mathrm{CH}_{2} \mathrm{Cl}_{2},-10^{\circ} \mathrm{C}, 4 \mathrm{~h}, 91 \%$ for $10,79 \%$ for $13,75 \%$ for $15,80 \%$ for 17 ; (f) thiourea, 2,4-lutidine, $\mathrm{CH}_{2} \mathrm{Cl}_{2}-\mathrm{MeOH}$, reflux, overnight, $89 \%$ for 12, $71 \%$ for 14; (g) satd $\mathrm{NH}_{3}-\mathrm{MeOH}$, rt, $48 \mathrm{~h}, 98 \%$.

EtOAc) indicated that the reaction was complete. The mixture was concentrated, and the residue so obtained was subjected to column chromatography with 3:1 petroleum ether-EtOAc as the eluent to give the product. 

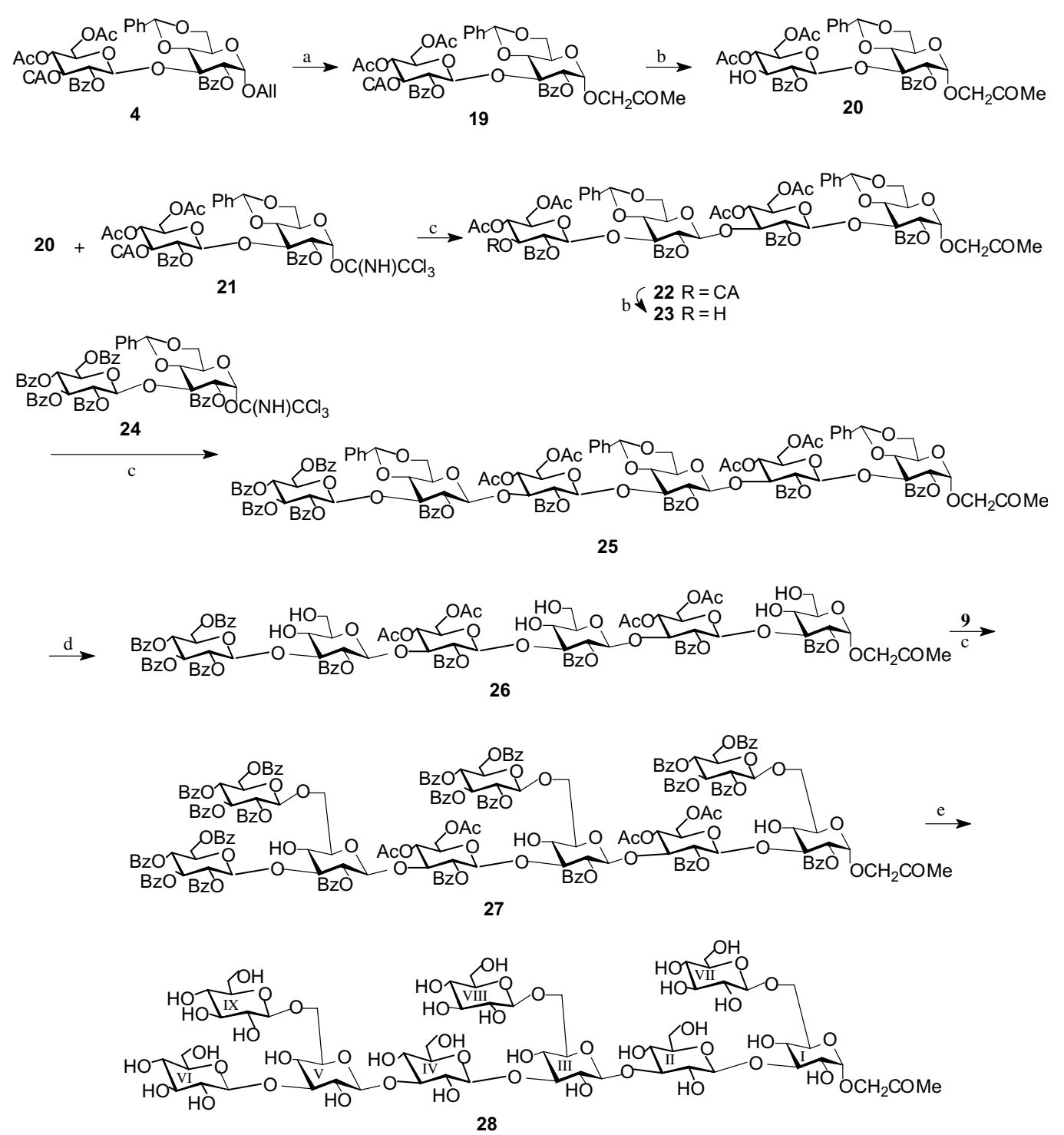

Scheme 2. Reagents and conditions: (a) $\mathrm{PdCl}_{2}$, THF, HOAc (75\%)-NaOAc, rt, overnight, $36 \%$; (b) thiourea, 2,4-lutidine, $\mathrm{CH}_{2} \mathrm{Cl}{ }_{2}-\mathrm{MeOH}_{\mathrm{O}}$, reflux, overnight, $87 \%$ for $\mathbf{2 0}, 81 \%$ for $\mathbf{2 3}$; (c) TMSOTf, $\mathrm{CH}_{2} \mathrm{Cl}_{2},-10{ }^{\circ} \mathrm{C}, 2-4 \mathrm{~h} ; 71 \%$ for $\mathbf{2 2}, 50 \%$ for $\mathbf{2 5}, 55 \%$ for $\mathbf{2 7}$; (d) $\mathrm{TsOH}_{2} \mathrm{CH}_{2}(\mathrm{OH})_{2}, \mathrm{MeCN}, \mathrm{rt}$, overnight, $69 \%$; (e) satd $\mathrm{NH}_{3}-\mathrm{MeOH}$, rt, $48 \mathrm{~h}, 98 \%$.

\subsection{General procedure for the coupling reaction}

To a solution of almost equivalent amounts of glycosyl acceptor and donor in $\mathrm{CH}_{2} \mathrm{Cl}_{2}(15-25 \mathrm{~mL})$ was added $4 \AA$ molecular sieves $(0.5-0.8 \mathrm{~g})$. The mixture was stirred and cooled to $-10^{\circ} \mathrm{C}$ under $\mathrm{N}_{2}$ protection, and TMSOTf (5-10\% equiv) was added. Stirring was continued at low temperature $\left(-10^{\circ} \mathrm{C}\right)$ for $4 \mathrm{~h}$, at the end of which time TLC (2:1 petroleum ether-EtOAc) indicated that the reaction was complete. $\mathrm{Et}_{3} \mathrm{~N}$ was added to stop the reaction, the mixture was filtered, and the filter cake was washed with $\mathrm{CH}_{2} \mathrm{Cl}_{2}$. The combined filtrate and washings were washed with satd aq $\mathrm{NaHCO}_{3}$ and water, then dried and concentrated. Purification by column chromatography with $3: 1$ petroleum ether-EtOAc as the eluent afforded the product.

\subsection{2-O-Benzoyl-4,6- $O$-benzylidene-3- $O$-chloroacetyl- $\alpha$ - D-glucopyranose (2)}

To a solution of $\mathrm{NaOAc}(980 \mathrm{mg}, 11.95 \mathrm{mmol})$ and $\mathrm{PdCl}_{2}(1.06 \mathrm{~g}, 5.98 \mathrm{mmol})$ in $\mathrm{HOAc}(75 \%, 5 \mathrm{~mL})$ was added compound $1(1.5 \mathrm{~g}, 3.07 \mathrm{mmol})$. The mixture was stirred at $\mathrm{rt}$ overnight under the $\mathrm{N}_{2}$ protection, at the end of which time TLC $(2: 1$ petroleum etherEtOAc) indicated that the reaction was complete. The mixture was filtered, and the filter cake was washed with $\mathrm{CH}_{2} \mathrm{Cl}_{2}$. The organic phase was washed with satd aq $\mathrm{NaHCO}_{3}$ and water, then dried and concentrated. Purification by column chromatography with $2: 1$ petroleum ether-EtOAc as the eluent afforded compound $2(1.1 \mathrm{~g}$, $80 \%)$ as a syrup: $[\alpha]_{\mathrm{D}}^{20}+90.0\left(c 0.7, \mathrm{CHCl}_{3}\right) ;{ }^{1} \mathrm{H}$ NMR: $\delta$ 8.04-7.36 (m, $10 \mathrm{H}, \mathrm{Bz}-H, \mathrm{Ph}-H), 5.90\left(\mathrm{dd}, 1 \mathrm{H}, J_{2,3}\right.$ 
$\left.9.8 \mathrm{~Hz}, J_{3,4} 9.8 \mathrm{~Hz}, \mathrm{H}-3\right), 5.65\left(\mathrm{~d}, 1 \mathrm{H}, J_{1,2} 3.7 \mathrm{~Hz}, \mathrm{H}-1\right)$, $5.55(\mathrm{~s}, 1 \mathrm{H}, \mathrm{PhCH}), 5.12\left(\mathrm{dd}, 1 \mathrm{H}, J_{1,2} 3.7 \mathrm{~Hz}, J_{2,3}\right.$ $9.8 \mathrm{~Hz}, \mathrm{H}-2), 4.33\left(\mathrm{dd}, 1 \mathrm{H}, J_{5,6 \mathrm{a}} 4.9 \mathrm{~Hz}, J_{6 \mathrm{a}, 6 \mathrm{~b}} 10.1 \mathrm{~Hz}\right.$, H-6a), 4.33-4.23 (m, 1H, H-5), 4.02, 3.97 (ABq, 2H, $J$ $\left.14.7 \mathrm{~Hz}, \mathrm{ClCH}_{2} \mathrm{CO}\right), 3.81\left(\mathrm{dd}, 1 \mathrm{H}, J_{5,6 \mathrm{~b}} 10.1 \mathrm{~Hz}, J_{6 \mathrm{a}, 6 \mathrm{~b}}\right.$ $10.1 \mathrm{~Hz}, \mathrm{H}-6 \mathrm{~b}), 3.80\left(\mathrm{dd}, 1 \mathrm{H}, J_{3,4} 9.8 \mathrm{~Hz}, J_{4,5} 9.8 \mathrm{~Hz}\right.$, H-4). Anal. Calcd for $\mathrm{C}_{22} \mathrm{H}_{21} \mathrm{ClO}_{8}$ : C, 58.87; H, 4.72 . Found: C, 58.76; H, 4.64.

\subsection{2-O-Benzoyl-4,6- $O$-benzylidene-3- $O$-chloroacetyl- $\alpha$ - D-glucopyranosyl trichloroacetimidate (3)}

Compound 3 was obtained as a syrup $(1.09 \mathrm{~g}, 82.5 \%)$ from compound $2(1.0 \mathrm{~g}, \quad 2.23 \mathrm{mmol}), \quad \mathrm{CCl}_{3} \mathrm{CN}$ $(0.66 \mathrm{~mL}, 6.6 \mathrm{mmol})$, and DBU $(55 \mu \mathrm{L})$ according to the general procedure for trichloroacetimidate formation: $[\alpha]_{\mathrm{D}}^{20}+78.0\left(c\right.$ 1.2, $\left.\mathrm{CHCl}_{3}\right) ;{ }^{1} \mathrm{H}$ NMR: $\delta 8.60(\mathrm{~s}$, $1 \mathrm{H}, \mathrm{N} H), 8.01-7.37(\mathrm{~m}, 10 \mathrm{H}, \mathrm{Bz}-H, \mathrm{Ph}-H), 6.71(\mathrm{~d}$, $\left.1 \mathrm{H}, J_{1,2} 3.8 \mathrm{~Hz}, \mathrm{H}-1\right), 5.94\left(\mathrm{dd}, 1 \mathrm{H}, J_{2,3}=J_{3,4}=9.8 \mathrm{~Hz}\right.$, $\mathrm{H}-3), 5.59(\mathrm{~s}, 1 \mathrm{H}, \mathrm{PhCH}), 5.40\left(\mathrm{dd}, 1 \mathrm{H}, J_{1,2} 3.8 \mathrm{~Hz}\right.$, $\left.J_{2,3} 9.8 \mathrm{~Hz}, \mathrm{H}-2\right), 4.41\left(\mathrm{dd}, 1 \mathrm{H}, J_{5,6 \mathrm{a}} 4.9 \mathrm{~Hz}, J_{6 \mathrm{a}, 6 \mathrm{~b}}\right.$ $10.4 \mathrm{~Hz}, \mathrm{H}-6 \mathrm{a}), 4.25-4.18$ (m, 1H, H-5), 4.03, 4.00 $\left(\mathrm{ABq}, 2 \mathrm{H}, \quad J 14.7 \mathrm{~Hz}, \mathrm{ClCH}_{2} \mathrm{CO}\right), 3.93(\mathrm{dd}, 1 \mathrm{H}$, $\left.J_{3,4}=J_{4,5}=9.8 \mathrm{~Hz}, \mathrm{H}-4\right), 3.85\left(\mathrm{dd}, 1 \mathrm{H}, J_{5,6} 10.4 \mathrm{~Hz}\right.$, $\left.J_{6 \mathrm{a}, 6 \mathrm{~b}} 10.4 \mathrm{~Hz}, \mathrm{H}-6\right)$. Anal. Calcd for $\mathrm{C}_{24} \mathrm{H}_{21} \mathrm{Cl}_{4} \mathrm{NO}_{8}$ : C, 48.59; H, 3.57. Found: C, 48.76; H, 3.62.

\subsection{Allyl 4,6-di- $O$-acetyl-2- $O$-benzoyl-3- $O$-chloroacetyl- $\beta$-D-glucopyranosyl-( $1 \rightarrow 3)-2-O$-benzoyl- $\alpha$-D-glucopyran- oside (5)}

To a solution of compounds $4(2 \mathrm{~g}, 2.38 \mathrm{mmol})$ and ethylene glycol $(1.0 \mathrm{~mL}, 17.97 \mathrm{mmol})$ in $\mathrm{MeCN}(20 \mathrm{~mL})$ was added $p$-toluenesulfonic acid $\cdot \mathrm{H}_{2} \mathrm{O}(50 \mathrm{mg})$. The mixture was stirred at $\mathrm{rt}$ overnight. TLC (2:1 petroleum etherEtOAc) indicated that the reaction was complete. The mixture was neutralized with $\mathrm{Et}_{3} \mathrm{~N}$, concentrated and extracted with $\mathrm{CH}_{2} \mathrm{Cl}_{2}$, and the organic phase was washed with water, then dried and concentrated. Purification by column chromatography with $1: 1$ petroleum ether-EtOAc as the eluent afforded compound $5(1.63 \mathrm{~g}, 91 \%)$ as a syrup: $[\alpha]_{\mathrm{D}}^{20}+87.5\left(c 1.0, \mathrm{CHCl}_{3}\right)$; ${ }^{1} \mathrm{H}$ NMR: $\delta$ 7.71-7.01 $(\mathrm{m}, 10 \mathrm{H}, 2 \mathrm{Bz}-H), 5.78-5.68$ (m, $\left.1 \mathrm{H}, \quad \mathrm{CH}_{2}=\mathrm{CH}-\mathrm{CH}_{2}-\right), \quad 5.44\left(\mathrm{dd}, \quad 1 \mathrm{H}, \quad J_{3,4}=\right.$ $\left.J_{2,3}=9.6 \mathrm{~Hz}, \mathrm{H}-3^{\prime}\right), 5.31\left(\mathrm{dd}, 1 \mathrm{H}, J_{1,2} 7.9 \mathrm{~Hz}, J_{2,3}\right.$ $\left.9.6 \mathrm{~Hz}, \mathrm{H}-2^{\prime}\right), \quad 5.20-5.05\left(\mathrm{~m}, 2 \mathrm{H}, \mathrm{CH}_{2}=\mathrm{CH}-\mathrm{CH}_{2}-\right)$, $5.17\left(\mathrm{dd}, 1 \mathrm{H}, J_{3,4}=J_{4,5}=9.6 \mathrm{~Hz}, \mathrm{H}-4^{\prime}\right), 5.10(\mathrm{~d}, 1 \mathrm{H}$, $\left.J_{1,2} 3.8 \mathrm{~Hz}, \mathrm{H}-1\right), 4.90\left(\mathrm{dd}, 1 \mathrm{H}, J_{1,2} 3.8 \mathrm{~Hz}, J_{2,3} 9.8 \mathrm{~Hz}\right.$, $\mathrm{H}-2), 4.88\left(\mathrm{~d}, 1 \mathrm{H}, J_{1,2} 7.9 \mathrm{~Hz}, \mathrm{H}-1^{\prime}\right), 4.28\left(\mathrm{~d}, 2 \mathrm{H}, J_{5,6}\right.$ $\left.4.0 \mathrm{~Hz}, \mathrm{H}-6^{\prime}\right), 4.16-3.76\left(\mathrm{~m}, 6 \mathrm{H}, \mathrm{CH}_{2}=\mathrm{CH}-\mathrm{CH}_{2-}, \mathrm{H}-5\right.$, $\left.5^{\prime}, 6\right), 3.85,3.78\left(\mathrm{ABq}, 2 \mathrm{H}, J 14.8 \mathrm{~Hz}, \mathrm{ClCH}_{2} \mathrm{CO}\right), 3.94$ $\left(\mathrm{dd}, \quad 1 \mathrm{H}, \quad J_{2,3}=J_{3,4}=9.8 \mathrm{~Hz}, \mathrm{H}-3\right), \quad 3.71(\mathrm{dd}, \quad 1 \mathrm{H}$, $\left.J_{3,4}=J_{4,5}=9.8 \mathrm{~Hz}, \mathrm{H}-4\right), 2.13,2.05\left(2 \mathrm{~s}, 6 \mathrm{H}, 2 \mathrm{CH}_{3} \mathrm{CO}\right)$. Anal. Calcd for $\mathrm{C}_{35} \mathrm{H}_{39} \mathrm{ClO}_{16}: \mathrm{C}, 55.96 ; \mathrm{H}, 5.23$. Found: C, 55.84; H, 5.12.
3.7. Allyl 4,6-di- $O$-acetyl-2- $O$-benzoyl-3- $O$-chloroacetyl$\beta$-D-glucopyranosyl-(1 $\rightarrow 3$ )-4,6-di- $O$-acetyl-2- $O$-benzoyl$\alpha$-D-glucopyranoside (6)

To a solution of compound $5(600 \mathrm{mg}, 0.80 \mathrm{mmol})$ in $\mathrm{CH}_{2} \mathrm{Cl}_{2}(10 \mathrm{~mL})$ was added pyridine $(4 \mathrm{~mL})$ and $\mathrm{Ac}_{2} \mathrm{O}$ $(3 \mathrm{~mL})$. The mixture was stirred at $\mathrm{rt}$ for $4 \mathrm{~h}$, at which time TLC (2:1 petroleum ether-EtOAc) indicated that the reaction was complete. Concentration of the mixture in vacuo and purification the residue by column chromatography with 3:1 petroleum ether-EtOAc as the eluent afforded compound $6(654 \mathrm{mg}, 98 \%)$ as a syrup: $[\alpha]_{\mathrm{D}}^{20}+55.9\left(c \quad 0.9, \mathrm{CHCl}_{3}\right)$; ${ }^{1} \mathrm{H}$ NMR: $\delta$ 7.89-7.07 (m, $10 \mathrm{H}, 2 \mathrm{Bz}-\mathrm{H}), \quad 5.80-5.70\left(\mathrm{~m}, 1 \mathrm{H}, \mathrm{CH}_{2}=\mathrm{CH}_{-} \mathrm{CH}_{2}-\right.$ ), $5.32\left(\mathrm{dd}, 1 \mathrm{H}, J_{3,4}=J_{2,3}=9.5 \mathrm{~Hz}, \mathrm{H}-3^{\prime}\right), 5.23-5.17(\mathrm{~m}$, $\left.3 \mathrm{H}, \mathrm{CH} H_{2}=\mathrm{CH}-\mathrm{CH}_{2}-, \mathrm{H}-2^{\prime}\right), 5.15\left(\mathrm{~d}, 1 \mathrm{H}, J_{1,2} 3.7 \mathrm{~Hz}\right.$, $\mathrm{H}-1), 5.12\left(\mathrm{dd}, 1 \mathrm{H}, J_{3,4}=J_{4,5}=9.8 \mathrm{~Hz}, \mathrm{H}-4^{\prime}\right), 5.09(\mathrm{dd}$, $\left.1 \mathrm{H}, J_{3,4}=J_{4,5}=9.5 \mathrm{~Hz}, \mathrm{H}-4\right), 4.91\left(\mathrm{~d}, 1 \mathrm{H}, J_{1,2} 7.7 \mathrm{~Hz}\right.$, H-1'), $4.88\left(\mathrm{dd}, 1 \mathrm{H}, J_{1,2} 3.7 \mathrm{~Hz}, J_{2,3} 9.8 \mathrm{~Hz}, \mathrm{H}-2\right), 4.47$ $\left(\mathrm{dd}, 1 \mathrm{H}, J_{5,6 \mathrm{a}}=4.2 \mathrm{~Hz}, J_{6 \mathrm{a}, 6 \mathrm{~b}} 12.4 \mathrm{~Hz}, \mathrm{H}-6 \mathrm{a}\right), 4.36(\mathrm{dd}$, $\left.1 \mathrm{H}, J_{2,3}=J_{3,4}=9.8 \mathrm{~Hz}, \quad \mathrm{H}-3\right), 4.21\left(\mathrm{dd}, 1 \mathrm{H}, J_{5,6 \mathrm{~b}}\right.$ $\left.4.5 \mathrm{~Hz}, \quad J_{6 \mathrm{a}, 6 \mathrm{~b}} 12.4 \mathrm{~Hz}, \quad \mathrm{H}-6 \mathrm{~b}\right), \quad 4.15-3.82 \quad(\mathrm{~m}, 6 \mathrm{H}$, $\left.\mathrm{CH}_{2}=\mathrm{CH}-\mathrm{CH}_{2-}, \mathrm{H}-5,5^{\prime}, 6^{\prime}\right), 3.84,3.78(\mathrm{ABq}, 2 \mathrm{H}, J$ $\left.14.9 \mathrm{~Hz}, \mathrm{ClCH}_{2} \mathrm{CO}\right), 2.11,2.11,2.07,2.04(4 \mathrm{~s}, 12 \mathrm{H}$, $4 \mathrm{CH}_{3} \mathrm{CO}$ ). Anal. Calcd for $\mathrm{C}_{39} \mathrm{H}_{43} \mathrm{ClO}_{18}: \mathrm{C}, 56.08 ; \mathrm{H}$, 5.19. Found: C, 56.16; H, 5.32.

\subsection{4,6-Di- $O$-acetyl-2- $O$-benzoyl-3- $O$-chloroacetyl- $\beta$-D- glucopyranosyl-(1 $\rightarrow 3$ )-4,6-di- $O$-acetyl-2- $O$-benzoyl- $\alpha$-D- glucopyranose (7)}

To a solution of compound $6(550 \mathrm{mg}, 0.66 \mathrm{mmol})$ in $\mathrm{MeOH}(10 \mathrm{~mL})$ was added $\mathrm{PdCl}_{2}(20 \mathrm{mg}, 0.11 \mathrm{mmol})$, and the mixture was stirred at $\mathrm{rt}$ for $8 \mathrm{~h}$, at the end of which time TLC (2:1 petroleum ether-EtOAc) indicated that the reaction was complete. The mixture was filtered, the filter cake was washed with $\mathrm{CH}_{2} \mathrm{Cl}_{2}$, and the combined filtrate and washings was concentrated. Purification by column chromatography with 2:1 petroleum ether-EtOAc as the eluent afforded compound 7 $(430 \mathrm{mg}, 82 \%)$ as a syrup: $[\alpha]_{\mathrm{D}}^{20}+30.8\left(\mathrm{c} 0.7, \mathrm{CHCl}_{3}\right)$; ${ }^{1} \mathrm{H}$ NMR: $\delta$ 7.90-7.08 (m, 10H, 2Bz-H), $5.54(\mathrm{br}$ $\mathrm{s}, 1 \mathrm{H}, \mathrm{H}-1), 5.33\left(\mathrm{dd}, 1 \mathrm{H}, J_{3,4}=J_{2,3}=9.5 \mathrm{~Hz}, \mathrm{H}-3^{\prime}\right)$, $5.22\left(\mathrm{dd}, 1 \mathrm{H}, \quad J_{1,2} \quad 7.8 \mathrm{~Hz}, J_{2,3} 9.5 \mathrm{~Hz}, \mathrm{H}-2^{\prime}\right), \quad 5.17$ $\left(\mathrm{dd}, 1 \mathrm{H}, \quad J_{3,4}=J_{4,5}=9.6 \mathrm{~Hz}, \quad \mathrm{H}-4^{\prime}\right), 5.09(\mathrm{dd}, 1 \mathrm{H}$, $\left.J_{3,4}=J_{4,5}=9.5 \mathrm{~Hz}, \mathrm{H}-4\right), 4.93\left(\mathrm{~d}, 1 \mathrm{H}, J_{1,2} 7.8 \mathrm{~Hz}, \mathrm{H}-\right.$ $\left.1^{\prime}\right), 4.88\left(\mathrm{dd}, 1 \mathrm{H}, J_{1,2} 3.6 \mathrm{~Hz}, J_{2,3} 9.6 \mathrm{~Hz}, \mathrm{H}-2\right), 4.47$ $\left(\mathrm{dd}, 1 \mathrm{H}, J_{5,6 \mathrm{a}} 4.2 \mathrm{~Hz}, J_{6 \mathrm{a}, 6 \mathrm{~b}} 12.5 \mathrm{~Hz}, \mathrm{H}-6 \mathrm{a}\right), 4.41(\mathrm{dd}$, $\left.1 \mathrm{H}, J_{2,3}=J_{3,4}=9.6 \mathrm{~Hz}, \mathrm{H}-3\right), 4.25-4.20\left(\mathrm{~m}, 1 \mathrm{H}, \mathrm{H}-5^{\prime}\right)$, $4.17\left(\mathrm{~d}, 2 \mathrm{H}, J_{5,6} 3.3 \mathrm{~Hz}, \mathrm{H}-6^{\prime}\right), 4.13\left(\mathrm{dd}, 1 \mathrm{H}, J_{5,6 \mathrm{~b}}\right.$ $\left.2.1 \mathrm{~Hz}, J_{6 \mathrm{a}, 6 \mathrm{~b}} 12.5 \mathrm{~Hz}, \mathrm{H}-6 \mathrm{~b}\right), 3.87-3.84(\mathrm{~m}, 1 \mathrm{H}, \mathrm{H}-5)$, $3.85,3.78\left(\mathrm{ABq}, 2 \mathrm{H}, J 14.9 \mathrm{~Hz}, \mathrm{ClCH}_{2} \mathrm{CO}\right), 2.11,2.11$, $2.08,2.04\left(4 \mathrm{~s}, 12 \mathrm{H}, 4 \mathrm{CH}_{3} \mathrm{CO}\right)$. Anal. Calcd for $\mathrm{C}_{36} \mathrm{H}_{39} \mathrm{ClO}_{18}$ : C, 54.38; H, 4.94. Found: C, 54.22; H, 4.78 . 
3.9. 4,6-Di- $O$-acetyl-2- $O$-benzoyl-3- $O$-chloroacetyl- $\beta$-Dglucopyranosyl-( $1 \rightarrow 3$ )-4,6-di- $O$-acetyl-2- $O$-benzoyl- $\alpha$-Dglucopyranosyl trichloroacetimidate $(8)$

Compound 8 was obtained as a syrup $(340 \mathrm{mg}, 87 \%$ ) from compound $7(330 \mathrm{mg}, \quad 0.42 \mathrm{mmol}), \quad \mathrm{CCl}_{3} \mathrm{CN}$ $(0.12 \mathrm{~mL}, 1.22 \mathrm{mmol})$, and $\mathrm{DBU}(10 \mu \mathrm{L})$ according to the general procedure for trichloroacetimidate formation: $[\alpha]_{\mathrm{D}}^{20}+54.3\left(c\right.$ 1.5, $\left.\mathrm{CHCl}_{3}\right) ;{ }^{1} \mathrm{H}$ NMR: $\delta 8.54(\mathrm{~s}$, $1 \mathrm{H}, \mathrm{N} H), 7.80-7.12(\mathrm{~m}, 10 \mathrm{H}, 2 \mathrm{Bz}-H), 6.55\left(\mathrm{~d}, 1 \mathrm{H}, J_{1,2}\right.$ $3.6 \mathrm{~Hz}, \mathrm{H}-1), 5.31\left(\mathrm{dd}, 1 \mathrm{H}, J_{3,4}=J_{2,3}=9.4 \mathrm{~Hz}, \mathrm{H}-3^{\prime}\right)$, 5.24-5.12 (m, 4H, H-2, 2', 4, 4'), $4.87\left(\mathrm{~d}, 1 \mathrm{H}, J_{1,2}\right.$ $\left.7.8 \mathrm{~Hz}, \mathrm{H}-1^{\prime}\right), 4.44\left(\mathrm{dd}, 1 \mathrm{H}, J_{5,6 \mathrm{a}} 4.5 \mathrm{~Hz}, J_{6 \mathrm{a}, 6 \mathrm{~b}} 12.5 \mathrm{~Hz}\right.$, $\mathrm{H}-6 \mathrm{a}), 4.38\left(\mathrm{dd}, 1 \mathrm{H}, J_{2,3}=J_{3,4}=9.7 \mathrm{~Hz}, \mathrm{H}-3\right), 4.23-$ $3.85\left(\mathrm{~m}, 5 \mathrm{H}, \mathrm{H}-5,5^{\prime}, 6 \mathrm{~b}, 6^{\prime}\right), 3.82,3.76(\mathrm{ABq}, 2 \mathrm{H}, J$ $\left.14.8 \mathrm{~Hz}, \mathrm{ClCH}_{2} \mathrm{CO}\right), 2.11,2.08,2.07,2.02(4 \mathrm{~s}, 12 \mathrm{H}$, $4 \mathrm{CH} 3 \mathrm{CO}$ ). Anal. Calcd for $\mathrm{C}_{38} \mathrm{H}_{39} \mathrm{Cl}_{4} \mathrm{NO}_{18}$ : C, 48.58; H, 4.18. Found: C, 48.26; H, 4.25.

3.10. Allyl 4,6-di- $O$-acetyl-2-O-benzoyl-3- $O$-chloroacetyl$\beta$-D-glucopyranosyl- $(1 \rightarrow 3)-[2,3,4,6$-tetra- $O$-benzoyl- $\beta$-Dglucopyranosyl-( $1 \rightarrow 6) \mid-2-O$-benzoyl- $\alpha$-D-glucopyranoside (10)

Compound 10 ( $806 \mathrm{mg}, 91 \%$ ) was obtained as an amorphous solid from compounds $5(500 \mathrm{mg}, 0.67 \mathrm{mmol})$ and $9(600 \mathrm{mg}, 0.80 \mathrm{mmol})$ according to the general procedure for the coupling reaction: $[\alpha]_{\mathrm{D}}^{20}+49.9$ (c 1.2 , $\left.\mathrm{CHCl}_{3}\right) ;{ }^{1} \mathrm{H}$ NMR: $\delta$ 8.04-6.98 (m, 30H, 6Bz- $\left.H\right), 5.93$ $\left(\mathrm{dd}, \quad 1 \mathrm{H}, \quad J_{2,3}=J_{3,4}=9.6 \mathrm{~Hz}, \mathrm{H}-3^{\prime \prime}\right), 5.70(\mathrm{dd}, 1 \mathrm{H}$, $\left.J_{3,4}=J_{4,5}=9.6 \mathrm{~Hz}, \mathrm{H}-4^{\prime \prime}\right), 5.57\left(\mathrm{dd}, 1 \mathrm{H}, J_{1,2} 7.9 \mathrm{~Hz}\right.$, $\left.J_{2,3} 9.6 \mathrm{~Hz}, \mathrm{H}-2^{\prime \prime}\right), 5.56-5.46\left(\mathrm{~m}, 1 \mathrm{H}, \mathrm{CH}_{2}=\mathrm{CH}-\mathrm{CH}_{2}-\right.$ ), $5.39\left(\mathrm{dd}, 1 \mathrm{H}, J_{3,4}=J_{2,3}=9.6 \mathrm{~Hz}, \mathrm{H}-3^{\prime}\right), 5.25(\mathrm{dd}, 1 \mathrm{H}$, $\left.J_{1,2} \quad 8.0 \mathrm{~Hz}, \quad J_{2,3} \quad 9.6 \mathrm{~Hz}, \quad \mathrm{H}-2^{\prime}\right), \quad 5.13 \quad(\mathrm{dd}, \quad 1 \mathrm{H}$, $\left.J_{3,4}=J_{4,5}=9.6 \mathrm{~Hz}, \quad \mathrm{H}-4^{\prime}\right), \quad 5.06-4.93 \quad(\mathrm{~m}, \quad 2 \mathrm{H}$, $\left.\mathrm{CH}_{2}=\mathrm{CH}-\mathrm{CH}_{2}-\right), 4.97\left(\mathrm{~d}, 1 \mathrm{H}, J_{1,2} 8.0 \mathrm{~Hz}, \mathrm{H}-1^{\prime}\right), 4.84$ $\left(\mathrm{d}, 1 \mathrm{H}, J_{1,2} 3.8 \mathrm{~Hz}, \mathrm{H}-1\right), 4.78\left(\mathrm{~d}, 1 \mathrm{H}, J_{1,2} 7.9 \mathrm{~Hz}, \mathrm{H}-\right.$ $\left.1^{\prime \prime}\right), 4.64\left(\mathrm{dd}, 1 \mathrm{H}, \mathrm{J}_{5,6 \mathrm{a}} 2.8 \mathrm{~Hz}, J_{6 \mathrm{a}, 6 \mathrm{~b}} 12.2 \mathrm{~Hz}, \mathrm{H}-6 \mathrm{a}^{\prime \prime}\right)$, $4.63\left(\mathrm{dd}, 1 \mathrm{H}, J_{1,2} 3.8 \mathrm{~Hz}, J_{2,3} 9.5 \mathrm{~Hz}, \mathrm{H}-2\right), 4.51(\mathrm{dd}$, $\left.1 \mathrm{H}, J_{5,6 \mathrm{~b}} 4.6 \mathrm{~Hz}, J_{6 \mathrm{a}, 6 \mathrm{~b}} 12.2 \mathrm{~Hz}, \mathrm{H}-6 \mathrm{~b}^{\prime \prime}\right), 4.31(\mathrm{bd}, 1 \mathrm{H}$, $\left.J_{6 \mathrm{a}, 6 \mathrm{~b}} 11.6 \mathrm{~Hz}, \mathrm{H}-6 \mathrm{a}\right), 4.22\left(\mathrm{~d}, 2 \mathrm{H}, J_{5,6} 4.0 \mathrm{~Hz}, \mathrm{H}-6^{\prime}\right)$, 4.22-3.72 (m, 5H, $\left.\mathrm{CH}_{2}=\mathrm{CH}-\mathrm{CH}_{2}-, \mathrm{H}-5,5^{\prime}, 5^{\prime \prime}\right), 3.99$ $\left(\mathrm{dd}, 1 \mathrm{H}, J_{2,3}=J_{3,4}=9.5 \mathrm{~Hz}, \mathrm{H}-3\right), 3.85,3.78(\mathrm{ABq}$, $\left.2 \mathrm{H}, J 14.8 \mathrm{~Hz}, \mathrm{ClCH}_{2} \mathrm{CO}\right), 3.54\left(\mathrm{dd}, 1 \mathrm{H}, J_{5,6 \mathrm{~b}} 4.7 \mathrm{~Hz}\right.$, $\left.J_{6 \mathrm{a}, 6 \mathrm{~b}} 11.6 \mathrm{~Hz}, \mathrm{H}-6 \mathrm{~b}\right), 3.44\left(\mathrm{dd}, 1 \mathrm{H}, J_{3,4}=J_{4,5}=9.5 \mathrm{~Hz}\right.$, $\mathrm{H}-4), 2.11,2.06\left(2 \mathrm{~s}, 6 \mathrm{H}, 2 \mathrm{CH}_{3} \mathrm{CO}\right)$. Anal. Calcd for $\mathrm{C}_{69} \mathrm{H}_{65} \mathrm{ClO}_{25}: \mathrm{C}, 62.32 ; \mathrm{H}, 4.93$. Found: $\mathrm{C}, 62.16 ; \mathrm{H}$, 4.80 .

3.11. Allyl 4,6-di- $O$-acetyl-2- $O$-benzoyl-3- $O$-chloroacetyl$\beta$-D-glucopyranosyl-( $\rightarrow 3$ )-[2,3,4,6-tetra- $O$-benzoyl- $\beta$-Dglucopyranosyl-( $1 \rightarrow 6)]-4-O$-acetyl-2- $O$-benzoyl- $\alpha$-Dglucopyranoside (11)

To a solution of compound $\mathbf{1 0}(700 \mathrm{mg}, 0.53 \mathrm{mmol})$ in $\mathrm{CH}_{2} \mathrm{Cl}_{2}(10 \mathrm{~mL})$ containing pyridine $(0.4 \mathrm{~mL})$ was cooled $-10^{\circ} \mathrm{C}$, was added a solution of $\mathrm{AcCl}(0.2 \mathrm{~mL}$, $2.80 \mathrm{mmol})$ in $\mathrm{CH}_{2} \mathrm{Cl}_{2}(5 \mathrm{~mL})$, and the mixture was stirred for $4 \mathrm{~h}$ at $\mathrm{rt}$. TLC (3:1 petroleum ether-EtOAc) indicated that the reaction was complete. The mixture was diluted with $\mathrm{CH}_{2} \mathrm{Cl}_{2}$ and poured into ice water, then the organic phase was separated, successively washed with $\mathrm{N} \mathrm{HCl}$, satd aq $\mathrm{NaHCO}_{3}$, and water, dried, and concentrated. Purification by column chromatography with 3:1 petroleum ether-EtOAc as the eluent afforded compound $11(650 \mathrm{mg}, 90 \%)$ as an amorphous solid: $[\alpha]_{\mathrm{D}}^{20}+31.9\left(c\right.$ 0.5, $\left.\mathrm{CHCl}_{3}\right) ;{ }^{1} \mathrm{H}$ NMR: $\delta$ 8.04-7.07 (m, $30 \mathrm{H}, 6 \mathrm{Bz}-H), 5.90\left(\mathrm{dd}, 1 \mathrm{H}, J_{2,3}=J_{3,4}=9.7 \mathrm{~Hz}, \mathrm{H}-3^{\prime \prime}\right)$, $5.67\left(\mathrm{dd}, 1 \mathrm{H}, J_{3,4}=J_{4,5}=9.7 \mathrm{~Hz}, \mathrm{H}-4^{\prime \prime}\right), 5.53(\mathrm{dd}, 1 \mathrm{H}$, $\left.J_{1,2} \quad 8.0 \mathrm{~Hz}, J_{2,3} 9.7 \mathrm{~Hz}, \mathrm{H}-2^{\prime \prime}\right), \quad 5.52-5.42(\mathrm{~m}, 1 \mathrm{H}$, $\left.\mathrm{CH}_{2}=\mathrm{CH}-\mathrm{CH}_{2}-\right), 5.27\left(\mathrm{dd}, 1 \mathrm{H}, J_{3,4}=J_{2^{\prime}, 3^{\prime}}=9.4 \mathrm{~Hz}\right.$, H-3'), $5.19\left(\mathrm{dd}, 1 \mathrm{H}, J_{1,2} 7.8 \mathrm{~Hz}, J_{2,3} 9.4 \mathrm{~Hz}, \mathrm{H}-2^{\prime}\right), 5.14$ $\left(\mathrm{dd}, 1 \mathrm{H}, J_{3,4}=J_{4,5}=9.4 \mathrm{~Hz}, \mathrm{H}-4^{\prime}\right), 5.03-4.93(\mathrm{~m}, 2 \mathrm{H}$, $\left.\mathrm{CH}_{2}=\mathrm{CH}-\mathrm{CH}_{2}-\right), 4.90\left(\mathrm{~d}, 1 \mathrm{H}, J_{1,2} 7.8 \mathrm{~Hz}, \mathrm{H}-1^{\prime}\right), 4.84$ $\left(\mathrm{d}, 1 \mathrm{H}, J_{1,2} 8.0 \mathrm{~Hz}, \mathrm{H}-1^{\prime \prime}\right), 4.80\left(\mathrm{~d}, 1 \mathrm{H}, J_{1,2} 3.7 \mathrm{~Hz}, \mathrm{H}-\right.$ 1), $4.79\left(\mathrm{dd}, 1 \mathrm{H}, J_{3,4}=J_{4,5}=9.8 \mathrm{~Hz}, \mathrm{H}-4\right), 4.70(\mathrm{dd}$, $\left.1 \mathrm{H}, J_{1,2} 3.7 \mathrm{~Hz}, J_{2.3} 9.5 \mathrm{~Hz}, \mathrm{H}-2\right), 4.63\left(\mathrm{dd}, 1 \mathrm{H}, J_{5,6 \mathrm{a}}\right.$ $\left.2.8 \mathrm{~Hz}, \quad J_{6 \mathrm{a}, 6 \mathrm{~b}}=12.2 \mathrm{~Hz}, \mathrm{H}-6 \mathrm{a}^{\prime \prime}\right), 4.48\left(\mathrm{dd}, 1 \mathrm{H}, J_{5,6 \mathrm{~b}}\right.$ $\left.4.5 \mathrm{~Hz}, \quad J_{6 \mathrm{a}, 6 \mathrm{~b}} 12.2 \mathrm{~Hz}, \quad \mathrm{H}-6 \mathrm{~b}^{\prime \prime}\right), \quad 4.46-3.37 \quad(\mathrm{~m}, 9 \mathrm{H}$, $\left.\mathrm{CH}_{2}=\mathrm{CH}-\mathrm{CH}_{2}-, \mathrm{H}-5,5^{\prime}, 5^{\prime \prime}, 6,6^{\prime}\right), 4.27(\mathrm{dd}, 1 \mathrm{H}$, $\left.J_{2,3}=J_{3,4}=9.8 \mathrm{~Hz}, \quad \mathrm{H}-3\right), \quad 3.83,3.76(\mathrm{ABq}, 2 \mathrm{H}, \quad J$ $\left.14.8 \mathrm{~Hz}, \mathrm{ClCH}_{2} \mathrm{CO}\right), 2.08,2.02,2.00\left(3 \mathrm{~s}, 9 \mathrm{H}, 3 \mathrm{CH}_{3} \mathrm{CO}\right)$. Anal. Calcd for $\mathrm{C}_{71} \mathrm{H}_{67} \mathrm{ClO}_{26}$ : C, 62.16; H, 4.92. Found: C, 61.86; H, 5.02.

3.12. Allyl 4,6-di- $O$-acetyl-2- $O$-benzoyl- $\beta$-D-glucopyranosyl-(1 $\rightarrow 3)-[2,3,4,6$-tetra- $O$-benzoyl- $\beta$-D-glucopyranosyl$(1 \rightarrow 6) \mid-4-O$-acetyl-2- $O$-benzoyl- $\alpha$-D-glucopyranoside (12)

Compound $10(550 \mathrm{mg}, 0.40 \mathrm{mmol})$ was dissolved in mixed solvents of $\mathrm{CH}_{2} \mathrm{Cl}_{2}(4 \mathrm{~mL})$ and $\mathrm{MeOH}(6 \mathrm{~mL})$. To the solution were added thiourea $(152 \mathrm{mg}$, $2.00 \mathrm{mmol})$ and $2,4-$ lutidine $(45 \mu \mathrm{L}, 0.41 \mathrm{mmol})$, and the reaction mixture was boiled under reflux for $16 \mathrm{~h}$, at the end of which time TLC (2:1 petroleum etherEtOAc) indicated that the reaction was complete. The mixture was concentrated and extracted with $\mathrm{CH}_{2} \mathrm{Cl}_{2}$, the organic phase was successively washed with $\mathrm{N}$ $\mathrm{HCl}$, satd aq $\mathrm{NaHCO}_{3}$, and water, dried, and concentrated. Purification by column chromatography with 2:1 petroleum ether-EtOAc as the eluent afforded $\mathbf{1 2}$ $(460 \mathrm{mg}, 89 \%)$ as an amorphous solid: $[\alpha]_{\mathrm{D}}^{20}+41.9(c$ 1.5, $\left.\mathrm{CHCl}_{3}\right) ;{ }^{1} \mathrm{H}$ NMR: $\delta 8.03-7.16(\mathrm{~m}, 30 \mathrm{H}, 6 \mathrm{Bz}-H)$, $5.90\left(\mathrm{dd}, 1 \mathrm{H}, J_{2,3}=J_{3,4}=9.7 \mathrm{~Hz}, \mathrm{H}-3^{\prime \prime}\right), 5.67(\mathrm{dd}, 1 \mathrm{H}$, $\left.J_{3,4}=J_{4,5}=9.7 \mathrm{~Hz}, \mathrm{H}-4^{\prime \prime}\right), 5.53\left(\mathrm{dd}, 1 \mathrm{H}, J_{1,2} 8.0 \mathrm{~Hz}\right.$, $\left.J_{2,3} 9.7 \mathrm{~Hz}, \mathrm{H}-2^{\prime \prime}\right), 5.55-5.45\left(\mathrm{~m}, 1 \mathrm{H}, \mathrm{CH}_{2}=\mathrm{CH}-\mathrm{CH}_{2}-\right.$ ), 5.06-4.98 (m, 4H, $\left.\mathrm{CH}_{2}=\mathrm{CH}-\mathrm{CH}_{2}-, \mathrm{H}-2^{\prime}, 4^{\prime}\right), 4.92(\mathrm{~d}$, $\left.1 \mathrm{H}, J_{1,2} 7.8 \mathrm{~Hz}, \mathrm{H}-1^{\prime}\right), 4.87\left(\mathrm{~d}, 1 \mathrm{H}, J_{1,2} 3.7 \mathrm{~Hz}, \mathrm{H}-1\right)$, $4.83\left(\mathrm{~d}, \quad 1 \mathrm{H}, \quad J_{1,2} \quad 8.0 \mathrm{~Hz}, \mathrm{H}-1^{\prime \prime}\right), 4.77(\mathrm{dd}, 1 \mathrm{H}$, $\left.J_{3,4}=J_{4,5}=9.6 \mathrm{~Hz}, \mathrm{H}-4\right), 4.74\left(\mathrm{dd}, 1 \mathrm{H}, J_{1,2} 3.7 \mathrm{~Hz}, J_{2.3}\right.$ $9.6 \mathrm{~Hz}, \mathrm{H}-2), 4.64\left(\mathrm{dd}, 1 \mathrm{H}, J_{5,6 \mathrm{a}} 2.9 \mathrm{~Hz}, J_{6 \mathrm{a}, 6 \mathrm{~b}} 12.3 \mathrm{~Hz}\right.$, H-6a" $), 4.48\left(\mathrm{dd}, 1 \mathrm{H}, J_{5,6 \mathrm{~b}} 4.7 \mathrm{~Hz}, J_{6 \mathrm{a}, 6 \mathrm{~b}} 12.3 \mathrm{~Hz}\right.$, 
$\left.\mathrm{H}-6 \mathrm{~b}^{\prime \prime}\right), 4.45-3.42\left(\mathrm{~m}, 9 \mathrm{H}, \mathrm{CH}_{2}=\mathrm{CH}-\mathrm{CH}_{2}-\mathrm{H}-5,5^{\prime}, 5^{\prime \prime}\right.$, $\left.6,6^{\prime}\right), 4.30\left(\mathrm{dd}, 1 \mathrm{H}, J_{2,3}=J_{3,4}=9.6 \mathrm{~Hz}, \mathrm{H}-3\right), 3.70(\mathrm{dd}$, $\left.1 \mathrm{H}, J_{2,3}=J_{3,4}=9.4 \mathrm{~Hz}, \mathrm{H}-3^{\prime}\right), 2.09,2.09,2.01,2.00$ (3s, 9H, 3C $\mathrm{H}_{3} \mathrm{CO}$ ). Anal. Calcd for $\mathrm{C}_{69} \mathrm{H}_{66} \mathrm{O}_{25}$ : C, 63.98; H, 5.14. Found: C, 63.76; H, 5.12.

\subsection{Allyl 2-O-benzoyl-4,6- $O$-benzylidene-3- $O$-chloro-} acetyl- $\beta$-D-glucopyranosyl- $(1 \rightarrow 3)-4,6$-di- $O$-acetyl-2- $O$ benzoyl- $\beta$-D-glucopyranosyl-( $1 \rightarrow 3)-[2,3,4,6$-tetra- $O$-benzoyl- $\beta$-D-glucopyranosyl- $(1 \rightarrow 6)]-4-O$-acetyl-2- $O$-benzoyl$\alpha$-D-glucopyranoside (13)

Compound 13 (380 mg, 79\%) was obtained as an amorphous solid from compounds $12(360 \mathrm{mg}, 0.28 \mathrm{mmol})$ and $3(200 \mathrm{mg}, 0.34 \mathrm{mmol})$ according to the general procedure for the coupling reaction: $[\alpha]_{\mathrm{D}}^{20}+13.5$ (c 0.5 , $\left.\mathrm{CHCl}_{3}\right)$; ${ }^{1} \mathrm{H}$ NMR: $\delta$ 8.10-7.00 $(\mathrm{m}, 40 \mathrm{H}, 7 \mathrm{Bz}-\mathrm{H}, \mathrm{Ph}-$ $H), 5.88\left(\mathrm{dd}, 1 \mathrm{H}, J_{2,3}=J_{3,4}=9.6 \mathrm{~Hz}, \mathrm{H}-3^{\mathrm{IV}}\right), 5.65(\mathrm{dd}$, $\left.1 \mathrm{H}, \quad J_{3,4}=J_{4,5}=9.6 \mathrm{~Hz}, \quad \mathrm{H}-4^{\mathrm{IV}}\right), 5.50 \quad\left(\mathrm{dd}, 1 \mathrm{H}, J_{1,2}\right.$ $\left.8.0 \mathrm{~Hz}, J_{2,3} 9.6 \mathrm{~Hz}, \mathrm{H}-2^{\mathrm{IV}}\right), 5.47(\mathrm{~s}, 1 \mathrm{H}, \mathrm{PhCH}), 5.48$ $5.38\left(\mathrm{~m}, \quad 1 \mathrm{H}, \quad \mathrm{CH}_{2}=\mathrm{CH}-\mathrm{CH}_{2}-\right), 5.21 \quad(\mathrm{dd}, \quad 1 \mathrm{H}$, $\left.J_{2,3}=J_{3,4}=9.2 \mathrm{~Hz}, \mathrm{H}-3^{\mathrm{III}}\right), 5.07\left(\mathrm{dd}, 1 \mathrm{H}, J_{1,2} 7.8 \mathrm{~Hz}\right.$, $\left.J_{2,3} 9.6 \mathrm{~Hz}, \mathrm{H}-2^{\mathrm{II}}\right), 5.02\left(\mathrm{dd}, 1 \mathrm{H}, J_{3,4}=J_{4,5}=9.6 \mathrm{~Hz}, \mathrm{H}-\right.$ $\left.4^{\mathrm{II}}\right), 5.02\left(\mathrm{dd}, 1 \mathrm{H}, J_{1,2} 8.0 \mathrm{~Hz}, J_{2,3} 9.2 \mathrm{~Hz}, \mathrm{H}-2^{\mathrm{III}}\right), 4.99$ $4.80\left(\mathrm{~m}, 2 \mathrm{H}, \quad \mathrm{CH}=\mathrm{CH}-\mathrm{CH}_{2}-\right), 4.87\left(\mathrm{~d}, 1 \mathrm{H}, J_{1,2}\right.$ $\left.8.0 \mathrm{~Hz}, \mathrm{H}-1^{\mathrm{IV}}\right), 4.70-4.60\left(\mathrm{~m}, 5 \mathrm{H}, \mathrm{H}-1^{\mathrm{I}-\mathrm{III}}, 2,4\right), 4.56$ $\left(\mathrm{dd}, 1 \mathrm{H}, J_{5,6} 3.1 \mathrm{~Hz}, J_{6,6} 12.4 \mathrm{~Hz}, \mathrm{H}-6^{\mathrm{IV}}\right), 4.47$ (dd, $1 \mathrm{H}$, $\left.J_{5,6} 4.7 \mathrm{~Hz}, J_{6,6^{\prime}} 12.4 \mathrm{~Hz}, \mathrm{H}-6^{\prime{ }^{\mathrm{IV}}}\right), 4.38\left(\mathrm{dd}, 1 \mathrm{H}, J_{5,6}\right.$ $\left.4.9 \mathrm{~Hz}, J_{6,6} 10.2 \mathrm{~Hz}, \mathrm{H}-6^{\mathrm{III}}\right), 4.33\left(\mathrm{dd}, 1 \mathrm{H}, J_{5,6} 4.2 \mathrm{~Hz}\right.$, $\left.J_{6,6^{\prime}} 12.4 \mathrm{~Hz}, \mathrm{H}-6^{\mathrm{II}}\right), 4.20-3.31\left(\mathrm{~m}, 13 \mathrm{H}, \mathrm{CH}_{2}=\mathrm{CH}-\right.$ $\left.\mathrm{CH}_{2}-, \mathrm{H}-3^{\mathrm{I}, \mathrm{II}}, 4^{\mathrm{III}}, 5^{\mathrm{I}-\mathrm{V}}, 6^{\mathrm{I}}, 6^{\mathrm{I}, \mathrm{II}, \mathrm{III}}\right), 3.83,3.76(\mathrm{ABq}$, $\left.2 \mathrm{H}, \mathrm{J} 14.8 \mathrm{~Hz}, \mathrm{ClCH}_{2} \mathrm{CO}\right), 2.08,2.02,1.94(3 \mathrm{~s}, 12 \mathrm{H}$, $\left.3 \mathrm{CH}_{3} \mathrm{CO}\right)$. Anal. Calcd for $\mathrm{C}_{91} \mathrm{H}_{85} \mathrm{ClO}_{32}: \mathrm{C}, 63.32 ; \mathrm{H}$, 4.96. Found: C, 63.26; H, 5.00.

3.14. Allyl 2- $O$-benzoyl-4,6- $O$-benzylidene- $\beta$-D-glucopyranosyl-( $1 \rightarrow 3)-4$,6-di- $O$-acetyl-2- $O$-benzoyl- $\beta$-D-glucopyranosyl-(1 $\rightarrow 3)$-[2,3,4,6-tetra- $O$-benzoyl- $\beta$-D-glucopyranosyl$(1 \rightarrow 6) \mid-4-O$-acetyl-2-O-benzoyl- $\alpha$-D-glucopyranoside (14)

Compound 13 (280 mg, 0.16 mmol) was dissolved in mixed solvents of $\mathrm{CH}_{2} \mathrm{Cl}_{2}(4 \mathrm{~mL})$ and $\mathrm{MeOH}(6 \mathrm{~mL})$. To the solution were added thiourea $(62 \mathrm{mg}, 0.82 \mathrm{mmol})$ and 2,4 -lutidine $(18 \mu \mathrm{L}, 0.16 \mathrm{mmol})$, and the reaction mixture was boiled under reflux for $16 \mathrm{~h}$, at the end of which time TLC (2:1 petroleum ether-EtOAc) indicated that the reaction was complete. The mixture was concentrated and extracted with $\mathrm{CH}_{2} \mathrm{Cl}_{2}$, the organic phase was washed with $\mathrm{N} \mathrm{HCl}$, satd aq $\mathrm{NaHCO}_{3}$, and water, dried, and concentrated. Purification by column chromatography with 2:1 petroleum ether-EtOAc as the eluent afforded $14(190 \mathrm{mg}, 71 \%)$ as an amorphous solid: $[\alpha]_{\mathrm{D}}^{20}+14.7\left(c\right.$ 1.2, $\left.\mathrm{CHCl}_{3}\right) ;{ }^{1} \mathrm{H}$ NMR: $\delta$ 8.02-7.30 (m, $40 \mathrm{H}, 7 \mathrm{Bz}-H, \mathrm{Ph}-H), 5.88\left(\mathrm{dd}, 1 \mathrm{H}, J_{2,3}=J_{3,4}=9.7 \mathrm{~Hz}\right.$, $\left.\mathrm{H}-3^{\mathrm{IV}}\right), 5.66\left(\mathrm{dd}, 1 \mathrm{H}, J_{3,4}=J_{4,5}=9.6 \mathrm{~Hz}, \mathrm{H}-4^{\mathrm{IV}}\right), 5.51$ $(\mathrm{s}, 1 \mathrm{H}, \mathrm{PhCH}), 5.51\left(\mathrm{dd}, 1 \mathrm{H}, J_{1,2} 8.0 \mathrm{~Hz}, J_{2,3} 9.7 \mathrm{~Hz}\right.$, $\left.\mathrm{H}-2^{\mathrm{IV}}\right), 5.50-5.40\left(\mathrm{~m}, 1 \mathrm{H}, \mathrm{CH}_{2}=\mathrm{CH}-\mathrm{CH}_{2}-\right), 5.10(\mathrm{dd}$, $\left.1 \mathrm{H}, \quad J_{3,4}=J_{4,5}=9.4 \mathrm{~Hz}, \quad \mathrm{H}-4^{\mathrm{II}}\right), \quad 5.04-4.87 \quad(\mathrm{~m}, \quad 4 \mathrm{H}$, $\left.\mathrm{CH}_{2}=\mathrm{CH}-\mathrm{CH}_{2}-, \mathrm{H}-1^{\mathrm{I}}, 2^{\mathrm{II}}\right), 4.88\left(\mathrm{~d}, 1 \mathrm{H}, J_{1.2} 8.0 \mathrm{~Hz}\right.$, $\left.\mathrm{H}-1^{\mathrm{IV}}\right), 4.73-4.55\left(\mathrm{~m}, 6 \mathrm{H}, \mathrm{H}-1^{\mathrm{II}, \mathrm{III}}, 2^{\mathrm{I}, \mathrm{III}}, 4,6^{\mathrm{IV}}\right), 4.50$ $3.33\left(\mathrm{~m}, 17 \mathrm{H}, \mathrm{CH}_{2}=\mathrm{CH}-\mathrm{CH}_{2}-, \mathrm{H}-3^{\mathrm{I}-\mathrm{III}}, 4^{\mathrm{III}}, 5^{\mathrm{I}-\mathrm{IV}}\right.$, $\left.6^{\prime \mathrm{IV}}, 6^{\mathrm{I}-\mathrm{III}}\right), 2.07,2.01,1.94\left(3 \mathrm{~s}, 12 \mathrm{H}, 3 \mathrm{CH}_{3} \mathrm{CO}\right)$. Anal. Calcd for $\mathrm{C}_{89} \mathrm{H}_{84} \mathrm{O}_{31}$ : C, 64.80; $\mathrm{H}$, 5.13. Found: C, 64.76; H, 5.02.

3.15. Allyl 4,6-di- $O$-acetyl-2- $O$-benzoyl-3- $O$-chloroacetyl$\beta$-D-glucopyranosyl-( $\rightarrow 3)-4,6$-di- $O$-acetyl-2- $O$-benzoyl$\beta$-D-glucopyranosyl- $(1 \rightarrow 3)-2-O$-benzoyl-4,6- $O$-benzylidene- $\beta$-D-glucopyranosyl-( $\rightarrow 3)-4$,6-di- $O$-acetyl-2- $O$-benzoyl- $\beta$-D-glucopyranosyl-( $\rightarrow 3)$-[2,3,4,6-tetra- $O$-benzoyl$\beta$-D-glucopyranosyl- $(1 \rightarrow 6)]-4-O$-acetyl-2- $O$-benzoyl- $\alpha$-Dglucopyranoside (15)

Compound 15 (110 mg, 75\%) was obtained as an amorphous solid from compounds 14 (100 $\mathrm{mg}, 0.061 \mathrm{mmol})$ and $8(75 \mathrm{mg}, 0.080 \mathrm{mmol})$ according to the general procedure for the coupling reaction. However, purification was carried out with $2: 1$ petroleum ether-EtOAc as the eluent: $[\alpha]_{\mathrm{D}}^{20}+66.0$ (c 1.2, $\left.\mathrm{CHCl}_{3}\right) ;{ }^{1} \mathrm{H}$ NMR: $\delta$ 8.02-7.05 (m, 50H, 9Bz-H, $\mathrm{Ph}-H), 5.87(\mathrm{dd}, 1 \mathrm{H}$, $\left.J_{2,3}=J_{3,4}=9.8 \mathrm{~Hz}, \quad \mathrm{H}-3^{\mathrm{VI}}\right), \quad 5.65 \quad\left(\mathrm{dd}, \quad 1 \mathrm{H}, \quad J_{3,4}=\right.$ $\left.J_{4,5}=9.8 \mathrm{~Hz}, \mathrm{H}-4^{\mathrm{VI}}\right), 5.50\left(\mathrm{dd}, 1 \mathrm{H}, J_{1,2} 8.0 \mathrm{~Hz}, J_{2,3}\right.$ $\left.9.8 \mathrm{~Hz}, \mathrm{H}-2^{\mathrm{VI}}\right), 5.47-5.37\left(\mathrm{~m}, 3 \mathrm{H}, \mathrm{CH}_{2}=\mathrm{CH}-\mathrm{CH}_{2}-\right.$, $\left.\mathrm{PhCH}, \mathrm{H}-3^{\mathrm{V}}\right), 5.07-4.78\left(\mathrm{~m}, 13 \mathrm{H}, \mathrm{CH}_{2}=\mathrm{CH}-\mathrm{CH}_{2}-\mathrm{H}-\right.$ $\left.1^{\mathrm{I}, \mathrm{VI}}, 2^{\mathrm{I}-\mathrm{V}}, 4^{\mathrm{I}, \mathrm{II}, \mathrm{IV}, \mathrm{V}}\right), 4.68-3.18\left(\mathrm{~m}, 29 \mathrm{H}, \mathrm{H}-1^{\mathrm{II}-\mathrm{V}}, 3^{\mathrm{I}-\mathrm{IV}}\right.$, $\left.4^{\mathrm{III}}, 5^{\mathrm{I}-\mathrm{VI}}, 6^{\mathrm{I}-\mathrm{VI}}\right), 3.77,3.71(\mathrm{ABq}, 2 \mathrm{H}, J 14.8 \mathrm{~Hz}$, $\left.\mathrm{ClCH}_{2} \mathrm{CO}\right), 2.05,2.01,1.96,1.91,1.91,1.91,1.91(7 \mathrm{~s}$, $\left.21 \mathrm{H}, \quad 7 \mathrm{CH}_{3} \mathrm{CO}\right) .{ }^{13} \mathrm{C}$ NMR $\left(100 \mathrm{MHz}, \mathrm{CDCl}_{3}\right): \delta$ $170.8,170.7,169.2,169.1,168.4,168.2,168.1$ (7C, $\left.7 \mathrm{CH}_{3} \mathrm{CO}-\right), 166.2,166.1,165.9,165.7,165.3,165.2$, 165.0, 164.9, 164.7 (9C, 9COPh), 134.3 (1C, $\left.\mathrm{CH}_{2}=\mathrm{CH}-\mathrm{CH}_{2}\right), 118.1\left(1 \mathrm{C}, \mathrm{CH}_{2}=\mathrm{CH}-\mathrm{CH}_{2}\right), 101.2$ (-CHPh), 101.0, 100.8, 100.7, 100.6, 99.9 (5C, $\beta \mathrm{C}-1)$, $95.8(1 \mathrm{C}, \alpha \mathrm{C}-1)$. Anal. Calcd for $\mathrm{C}_{125} \mathrm{H}_{121} \mathrm{ClO}_{48}: \mathrm{C}$, 61.86; H, 5.03. Found: C, 61.95; H, 5.08.

3.16. Allyl 4,6-di- $O$-acetyl-2- $O$-benzoyl-3- $O$-chloroacetyl$\beta$-D-glucopyranosyl-( $\longrightarrow 3$ )-4,6-di- $O$-acetyl-2- $O$-benzoyl$\beta$-D-glucopyranosyl-( $1 \rightarrow 3)-2$ - $O$-benzoyl- $\beta$-D-glucopyranosyl-( $\rightarrow 3)-4,6$-di- $O$-acetyl-2- $O$-benzoyl- $\beta$-D-glucopyranosyl-( $1 \rightarrow 3)$-[2,3,4,6-tetra- $O$-benzoyl- $\beta$-D-glucopyranosyl$(1 \rightarrow 6)]-4-O$-acetyl-2- $O$-benzoyl- $\alpha$-D-glucopyranoside (16)

To a solution of compounds $15(70 \mathrm{mg}, 0.029 \mathrm{mmol})$ and ethylene glycol $(0.1 \mathrm{~mL}, 1.80 \mathrm{mmol})$ in $\mathrm{MeCN}(5 \mathrm{~mL})$ was added $p$-toluenesulfonic acid $\cdot \mathrm{H}_{2} \mathrm{O}(10 \mathrm{mg})$. The mixture was stirred at $\mathrm{rt}$ overnight, at the end of which time TLC (1:1 petroleum ether-EtOAc) indicated that the reaction was complete. The mixture was made neutral with $\mathrm{Et}_{3} \mathrm{~N}$, concentrated and extracted with $\mathrm{CH}_{2} \mathrm{Cl}_{2}$ and the organic phase was washed with water, then 
dried and concentrated. Purification by column chromatography with 1:1 petroleum ether-EtOAc as the eluent afforded compound $\mathbf{1 6}(53 \mathrm{mg}, 79 \%)$ as an amorphous solid: $[\alpha]_{\mathrm{D}}^{20}+6.4\left(c\right.$ 1.2, $\left.\mathrm{CHCl}_{3}\right) ;{ }^{1} \mathrm{H}$ NMR: $\delta$ 8.01-7.11 $(\mathrm{m}, 45 \mathrm{H}, 9 \mathrm{Bz}-H), 5.87\left(\mathrm{dd}, 1 \mathrm{H}, J_{2,3}=J_{3,4}=9.7 \mathrm{~Hz}, \mathrm{H}-\right.$ $\left.3^{\mathrm{VI}}\right), \quad 5.65\left(\mathrm{dd}, 1 \mathrm{H}, J_{3,4}=J_{4,5}=9.7 \mathrm{~Hz}, \mathrm{H}-4^{\mathrm{VI}}\right), 5.49$ $\left(\mathrm{dd}, 1 \mathrm{H}, J_{1,2} 8.0 \mathrm{~Hz}, J_{2,3} 9.7 \mathrm{~Hz}, \mathrm{H}-2^{\mathrm{VI}}\right), 5.46-5.37(\mathrm{~m}$, $\left.1 \mathrm{H}, \mathrm{CH}_{2}=\mathrm{CH}-\mathrm{CH}_{2}-\right), 5.09-3.14\left(\mathrm{~m}, 43 \mathrm{H}, \mathrm{CH}_{2}=\mathrm{CH}-\right.$ $\left.\mathrm{CH}_{2-}, \mathrm{H}-1^{\mathrm{I}-\mathrm{VI}}, 2^{\mathrm{II}-\mathrm{V}}, 3^{\mathrm{I}-\mathrm{V}}, 4^{\mathrm{I}-\mathrm{V}}, 5^{\mathrm{I}-\mathrm{VI}}, 6^{\mathrm{I}-\mathrm{VI}}\right), 3.77,3.71$ (ABq, $2 \mathrm{H}, J 14.8 \mathrm{~Hz}, \mathrm{ClCH}_{2} \mathrm{CO}$ ), 2.06, 2.05, 2.03, 1.98, $1.97,1.90,1.84\left(7 \mathrm{~s}, 21 \mathrm{H}, 7 \mathrm{CH}_{3} \mathrm{CO}\right)$. Anal. Calcd for $\mathrm{C}_{118} \mathrm{H}_{117} \mathrm{ClO}_{48}: \mathrm{C}, 60.60 ; \mathrm{H}, 5.04$. Found: $\mathrm{C}, 60.72 ; \mathrm{H}$, 5.12 .

3.17. Allyl 4,6-di- $O$-acetyl-2- $O$-benzoyl-3- $O$-chloroacetyl$\beta$-D-glucopyranosyl-( $\rightarrow 3)-4,6$-di- $O$-acetyl-2- $O$-benzoyl$\beta$-D-glucopyranosyl-( $1 \rightarrow 3)-[2,3,4,6$-tetra- $O$-benzoyl- $\beta$-Dglucopyranosyl-(1 $\rightarrow 6)]$-2- $O$-benzoyl- $\beta$-D-glucopyranosyl$(1 \rightarrow 3)-4,6$-di- $O$-acetyl-2- $O$-benzoyl- $\beta$-D-glucopyranosyl$(1 \rightarrow 3)$-[2,3,4,6-tetra- $O$-benzoyl- $\beta$-D-glucopyranosyl$(1 \rightarrow 6) \mid-4-O$-acetyl-2- $O$-benzoyl- $\alpha$-D-glucopyranoside (17)

Compound 17 (30 mg, 80\%) was obtained as an amorphous solid from compounds $\mathbf{1 6}(30 \mathrm{mg}, 0.013 \mathrm{mmol})$ and $9(12 \mathrm{mg}, 0.16 \mathrm{mmol})$ according to the general procedure for the coupling reaction. However, purification was carried out with 1:1 petroleum ether-EtOAc as the eluent: $[\alpha]_{\mathrm{D}}^{20}+19.8\left(c \quad 0.1, \mathrm{CHCl}_{3}\right) ;{ }^{1} \mathrm{H}$ NMR: $\delta$ 8.02-7.08 (m, 65H, 13Bz-H), 5.93, $5.87(\mathrm{dd}, 2 \mathrm{H}$, $\left.J_{2,3}=J_{3,4}=9.8 \mathrm{~Hz}, \quad \mathrm{H}-3^{\mathrm{VI}, \mathrm{VII}}\right), 5.74,5.64(\mathrm{dd}, 1 \mathrm{H}$, $\left.J_{3,4}=J_{4,5}=9.8 \mathrm{~Hz}, \mathrm{H}-4^{\mathrm{VI}, \mathrm{VII}}\right), 5.57-5.35\left(\mathrm{~m}, 3 \mathrm{H}, J_{1,2}\right.$ $\left.8.0 \mathrm{~Hz}, J_{2,3} 9.8 \mathrm{~Hz}, \mathrm{CH}_{2}=\mathrm{CH}-\mathrm{CH}_{2}-, \mathrm{H}-2^{\mathrm{VI}, \mathrm{VII}}\right), 5.16-$ $3.18\left(\mathrm{~m}, 47 \mathrm{H}, \mathrm{CH}_{2}=\mathrm{CH}-\mathrm{CH}_{2^{-}}, \mathrm{H}-1^{\mathrm{I}-\mathrm{VII}}, 2^{\mathrm{I}-\mathrm{V}}, 3^{\mathrm{I}-\mathrm{V}}\right.$, $\left.4^{\mathrm{I}-\mathrm{V}}, 5^{\mathrm{I}-\mathrm{VII}}, 6^{\mathrm{I}-\mathrm{VII}}\right), 3.72,3.66(\mathrm{ABq}, 2 \mathrm{H}, J 14.8 \mathrm{~Hz}$, $\left.\mathrm{ClCH}_{2} \mathrm{CO}\right), 2.05,2.04,2.00,1.99,1.96,1.86,1.77(7 \mathrm{~s}$, $\left.21 \mathrm{H},{ }_{7} \mathrm{CH}_{3} \mathrm{CO}\right) .{ }^{13} \mathrm{C}$ NMR: $\delta 170.6,170.6,170.4$, $170.2,169.8,169.3,169.2\left(7 \mathrm{C}, 7 \mathrm{CH}_{3} \mathrm{CO}\right), 166.1,165.9$, $165.7,165.5,165.2,165.2,165.1,164.9,164.8,164.7$, $163.7,163.6,163.4(13 \mathrm{C}, 13 \mathrm{BzCO}), 117.30$ (s, 1C, $\left.\mathrm{CH}_{2}=\mathrm{CH}-\mathrm{CH}_{2}\right), \quad 102.0, \quad 101.6,101.3,101.2,100.8$, 100.6 (6C, $\beta-\mathrm{C}-1), 94.3$ (1C, $\alpha-\mathrm{C}-1), 85.4,84.5,83.4$, 82.3 (4C, 4C-3). Anal. Calcd for $\mathrm{C}_{152} \mathrm{H}_{143} \mathrm{ClO}_{57}$ : C, 62.58; H, 4.94. Found: C, 62.72; H, 5.02.

3.18. Allyl $\beta$-D-glucopyranosyl-( $\rightarrow 3)-\beta$-D-glucopyranosyl-( $1 \rightarrow 3)$-[ $\beta$-D-glucopyranosyl-( $(1 \rightarrow 6)]-\beta$-D-glucopyranosyl-( $1 \rightarrow 3)-\beta$-D-glucopyranosyl-( $(1 \rightarrow 3)$-[ $\beta$-D-glucopyranosyl-(1 $\rightarrow 6)]-\alpha$-D-glucopyranoside $(18)$

Satd $\mathrm{NH}_{3}$ in $\mathrm{MeOH}(5 \mathrm{~mL})$ was added to compound $\mathbf{1 7}$ $(30 \mathrm{mg}, 10.3 \mu \mathrm{mol})$ in $\mathrm{MeOH}(4 \mathrm{~mL})$. After $48 \mathrm{~h}$ at rt, the reaction mixture was concentrated, and the residue was purified by Sephadex LH-20 chromatography $(\mathrm{MeOH})$ to afford $18(12 \mathrm{mg}, 98 \%)$ as an amorphous solid: $[\alpha]_{\mathrm{D}}^{20}+2.0\left(c \quad 0.4, \mathrm{H}_{2} \mathrm{O}\right) ;{ }^{1} \mathrm{H}$ NMR $\left(\mathrm{D}_{2} \mathrm{O}\right): \delta 6.05-5.95$ (m, $\left.\quad 1 \mathrm{H}, \quad \mathrm{CH}_{2}=\mathrm{C} H-\mathrm{CH}_{2}-\right), \quad 5.40-5.24 \quad(\mathrm{~m}, \quad 2 \mathrm{H}$,
$\left.\mathrm{CH}_{2}=\mathrm{CH}-\mathrm{CH}_{2}-\right), 4.96\left(\mathrm{~d}, 1 \mathrm{H}, J_{1,2} 3.6 \mathrm{~Hz}, \mathrm{H}-1^{\mathrm{I}}\right), 4.84$, $4.81,4.75,4.74\left(4 \mathrm{~d}, 4 \mathrm{H}, J_{1,2} 8.0 \mathrm{~Hz}, \mathrm{H}-1\right), 4.51,4.48$ $\left(2 \mathrm{~d}, \quad 2 \mathrm{H}, \quad J_{1,2} \quad 8.0 \mathrm{~Hz}, \mathrm{H}-1\right), \quad 4.28-3.28(\mathrm{~m}, \quad 44 \mathrm{H}$, $\left.\mathrm{CH}_{2}=\mathrm{CH}-\mathrm{CH}_{2}-, \mathrm{H}-2^{\mathrm{I}-\mathrm{VII}}, 3^{\mathrm{I}-\mathrm{VII}}, 4^{\mathrm{I}-\mathrm{VII}}, 5^{\mathrm{I}-\mathrm{VII}}, 6^{\mathrm{I}-\mathrm{VII}}\right)$. ${ }^{13} \mathrm{C}$ NMR $\left(\mathrm{D}_{2} \mathrm{O}\right): \delta 133.2\left(\mathrm{~s}, 1 \mathrm{C}, \mathrm{CH}_{2}=\mathrm{CH}-\mathrm{CH}_{2}\right)$, 118.1 (s, $1 \mathrm{C}, \mathrm{CH}_{2}=\mathrm{CH}-\mathrm{CH}_{2}$ ), 102.4, 102.3, 102.3, 102.2, 102.2, 102.1 (6C, $\beta-\mathrm{C}-1), 94.1$ (1C, $\alpha-\mathrm{C}-1), 84.2$, 84.1, 83.9, 83.7, 75.6, 75.2, 75.2, 73.1, 72.9, 72.8, 72.4, 69.2, 67.7, 60.4, 60.3, 60.2, (C-2 to C-6). Anal. Calcd for $\mathrm{C}_{45} \mathrm{H}_{76} \mathrm{O}_{36}: \mathrm{C}, 45.30 ; \mathrm{H}, 6.42$. Found: $\mathrm{C}, 45.16 ; \mathrm{H}$, 6.32 .

3.19. Acetonyl 4,6-di- $O$-acetyl-2- $O$-benzoyl-3- $O$-chloroacetyl- $\beta$-D-glucopyranosyl-( $1 \rightarrow 3)-2-O$-benzoyl-4,6- $O$ benzylidene- $\alpha$-D-glucopyranoside (19)

To a solution of compound $4(1.2 \mathrm{~g}, 1.48 \mathrm{mmol})$ in THF $(5 \mathrm{~mL})$ was added $\mathrm{HOAc}(75 \%, 5 \mathrm{~mL})$ containing $\mathrm{NaOAc}(500 \mathrm{mg}, 6.10 \mathrm{mmol})$, then $\mathrm{PdCl}_{2}(525 \mathrm{mg}$, $2.96 \mathrm{mmol}$ ) was added. The mixture was stirred at $\mathrm{rt}$ overnight, and TLC (2:1 petroleum ether-EtOAc) indicated that the reaction was complete. The mixture was filtered, the filter cake was washed with $\mathrm{CH}_{2} \mathrm{Cl}_{2}$, and the combined filtrate and washings were concentrated. Purification by column chromatography with $2: 1$ petroleum ether-EtOAc as the eluent afforded compound $\mathbf{1 9}$ $(440 \mathrm{mg}, 36 \%)$ as a syrup: $[\alpha]_{\mathrm{D}}^{20}+32.4\left(c 0.8, \mathrm{CHCl}_{3}\right)$; ${ }^{1} \mathrm{H}$ NMR: $\delta$ 7.82-7.13 (m, $\left.15 \mathrm{H}, 2 \mathrm{Bz}-H, \mathrm{Ph}-H\right), 5.61$ $(\mathrm{s}, 1 \mathrm{H}, \mathrm{PhCH}), 5.34\left(\mathrm{dd}, 1 \mathrm{H}, J_{2,3}=J_{3,4}=9.6 \mathrm{~Hz}, \mathrm{H}-\right.$ $\left.3^{\prime}\right), 5.29\left(\mathrm{dd}, 1 \mathrm{H}, J_{1,2} 7.8 \mathrm{~Hz}, J_{2,3} 9.6 \mathrm{~Hz}, \mathrm{H}-2^{\prime}\right), 5.17$ $\left(\mathrm{dd}, 1 \mathrm{H}, J_{3,4}=J_{4,5}=9.8 \mathrm{~Hz}, \mathrm{H}-4^{\prime}\right), 5.13\left(\mathrm{~d}, 1 \mathrm{H}, J_{1,2}\right.$ $3.6 \mathrm{~Hz}, \mathrm{H}-1), 5.01\left(\mathrm{dd}, 1 \mathrm{H}, J_{1,2} 3.6 \mathrm{~Hz}, J_{2,3} 9.8 \mathrm{~Hz}\right.$, $\mathrm{H}-2), 4.98\left(\mathrm{~d}, 1 \mathrm{H}, J_{1,2} 7.8 \mathrm{~Hz}, \mathrm{H}-1^{\prime}\right), 4.67(\mathrm{dd}, 1 \mathrm{H}$, $\left.J_{2,3}=J_{3,4}=9.8 \mathrm{~Hz}, \mathrm{H}-3\right), 4.27-3.67\left(\mathrm{~m}, 7 \mathrm{H}, \mathrm{H}-4,5,5^{\prime}\right.$, $\left.6,6^{\prime}\right), 4.15,4.00\left(\mathrm{ABq}, 2 \mathrm{H}, J 17.3 \mathrm{~Hz}, \mathrm{CH}_{2} \mathrm{COCH}_{3}\right)$, $3.83,3.77\left(\mathrm{ABq}, 2 \mathrm{H}, J 14.8 \mathrm{~Hz}, \mathrm{ClCH}_{2} \mathrm{CO}\right), 2.01,2.01$, $2.00\left(3 \mathrm{~s}, 9 \mathrm{H}, 3 \mathrm{CH}_{3} \mathrm{CO}\right)$. Anal. Calcd for $\mathrm{C}_{42} \mathrm{H}_{43} \mathrm{ClO}_{17}$ : C, 58.98; H, 5.07. Found: C, 59.06; H, 5.12.

\subsection{Acetonyl 4,6-di- $O$-acetyl-2- $O$-benzoyl- $\beta$-D-gluco- pyranosyl-( $1 \rightarrow 3)-2-O$-benzoyl-4,6- $O$-benzylidene- $\alpha-\mathrm{D}-$ glucopyranoside (20)}

Compound 19 ( $400 \mathrm{mg}, 0.485 \mathrm{mmol})$ was dissolved in mixed solvents of $\mathrm{CH}_{2} \mathrm{Cl}_{2}(4 \mathrm{~mL})$ and $\mathrm{MeOH}(6 \mathrm{~mL})$. To the solution were added thiourea $(185 \mathrm{mg}$, $2.43 \mathrm{mmol}$ ) and $2,4-$ lutidine $(54 \mu \mathrm{L}, 0.49 \mathrm{mmol})$, and the reaction mixture was boiled under reflux for $16 \mathrm{~h}$, at the end of which time TLC (2:1 petroleum etherEtOAc) indicated that the reaction was complete. The mixture was concentrated, and extracted with $\mathrm{CH}_{2} \mathrm{Cl}_{2}$, and the organic phase was washed with $\mathrm{N} \mathrm{HCl}$, satd aq $\mathrm{NaHCO}_{3}$, and water, dried and concentrated. Purification by column chromatography with $2: 1$ petroleum ether-EtOAc as the eluent afforded $20(330 \mathrm{mg}, 87 \%)$ as an amorphous solid: $[\alpha]_{\mathrm{D}}^{20}+50.4\left(c 1.0, \mathrm{CHCl}_{3}\right) ;{ }^{1} \mathrm{H}$ 
NMR: $\delta$ 7.92-7.21 (m, 15H, 2Bz-H, Ph-H), $5.60(\mathrm{~s}, 1 \mathrm{H}$, $\mathrm{PhCH}$ ), 5.17 (d, 1H, $\left.J_{1,2} 3.9 \mathrm{~Hz}, \mathrm{H}-1\right), 5.09$ (dd, 1H, $J_{1,2}$ $\left.7.8 \mathrm{~Hz}, J_{2,3} 9.4 \mathrm{~Hz}, \mathrm{H}-2^{\prime}\right), 5.03\left(\mathrm{dd}, 1 \mathrm{H}, J_{1,2} 3.9 \mathrm{~Hz}, J_{2,3}\right.$ $9.6 \mathrm{~Hz}, \mathrm{H}-2), 5.01\left(\mathrm{dd}, 1 \mathrm{H}, J_{3,4}=J_{4,5}=9.4 \mathrm{~Hz}, \mathrm{H}-4^{\prime}\right)$, $4.98\left(\mathrm{~d}, \quad 1 \mathrm{H}, \quad J_{1,2} \quad 7.8 \mathrm{~Hz}, \mathrm{H}-1^{\prime}\right), 4.51 \quad(\mathrm{dd}, 1 \mathrm{H}$, $\left.J_{2,3}=J_{3,4}=9.6 \mathrm{~Hz}, \mathrm{H}-3\right), 4.27-3.60$ (m, 8H, H-3', 4, 5, $\left.5^{\prime}, 6,6^{\prime}\right), 4.16,4.05\left(\mathrm{ABq}, 2 \mathrm{H}, J 17.3 \mathrm{~Hz}, \mathrm{CH}_{2} \mathrm{COCH}_{3}\right)$, 2.07, 2.01, $2.01\left(3 \mathrm{~s}, 9 \mathrm{H}, 3 \mathrm{CH}_{3} \mathrm{CO}\right)$. Anal. Calcd for $\mathrm{C}_{40} \mathrm{H}_{42} \mathrm{O}_{16}$ : C, 61.69; H, 5.44. Found: C, 61.76; H, 5.34.

3.21. Acetonyl 4,6-di- $O$-acetyl-2- $O$-benzoyl-3- $O$-chloroacetyl- $\beta$-D-glucopyranosyl-( $\longrightarrow 3)$-2- $O$-benzoyl-4,6- $O$ benzylidene- $\beta$-D-glucopyranosyl-( $(\longrightarrow 3)-4,6$-di- $O$-acetyl-2$O$-benzoyl- $\beta$-D-glucopyranosyl- $(1 \rightarrow 3)-2-O$-benzoyl-4,6$O$-benzylidene- $\alpha$-D-glucopyranoside (22)

Compound 22 (400 mg, 71\%) was obtained as an amorphous solid from compounds $20(280 \mathrm{mg}, 0.36 \mathrm{mmol})$ and 21 (400 mg, $0.43 \mathrm{mmol}$ ) according to the general procedure for the coupling reaction. However, purification by column chromatography was carried out with 2:1 petroleum ether-EtOAc as the eluent: $[\alpha]_{\mathrm{D}}^{20}+11.1(c$ 1.2, $\left.\mathrm{CHCl}_{3}\right) ;{ }^{1} \mathrm{H}$ NMR: $\delta$ 7.79-7.13 (m, 30H, $4 \mathrm{Bz}-H$, $2 \mathrm{Ph}-H), 5.52,5.48(2 \mathrm{~s}, 2 \mathrm{H}, 2 \mathrm{PhCH}), 5.12-4.92(\mathrm{~m}$, $\left.6 \mathrm{H}, \mathrm{H}-2^{\mathrm{II}-\mathrm{IV}}, 3^{\mathrm{IV}}, 4^{\mathrm{II}, \mathrm{IV}}\right), 5.05\left(\mathrm{~d}, 1 \mathrm{H}, \mathrm{d}, 1 \mathrm{H}, J_{1,2}\right.$ $\left.3.6 \mathrm{~Hz}, \mathrm{H}-1^{\mathrm{I}}\right), 4.79,4.64,4.60\left(3 \mathrm{~d}, 3 \mathrm{H}, J_{1,2} 7.8 \mathrm{~Hz}, \mathrm{H}-\right.$ $\left.1^{\mathrm{II}-\mathrm{IV}}\right), 4.71\left(\mathrm{dd}, 1 \mathrm{H}, J_{1,2} 3.6 \mathrm{~Hz}, J_{2,3} 9.6 \mathrm{~Hz}, \mathrm{H}-2^{\mathrm{I}}\right)$, $4.36-3.30\left(\mathrm{~m}, 17 \mathrm{H}, \mathrm{H}-3^{\mathrm{I}-\mathrm{III}}, 4^{\mathrm{I} \text { IIII }}, 5^{\mathrm{I}-\mathrm{IV}}, 6^{\mathrm{I}-\mathrm{IV}}\right), 4.08$, $3.98\left(\mathrm{ABq}, 2 \mathrm{H}, J=17.3 \mathrm{~Hz}, \mathrm{CH}_{2} \mathrm{COCH}_{3}\right), 3.75,3.68$ (ABq, 2H, $\left.J 14.8 \mathrm{~Hz}, \mathrm{ClCH}_{2} \mathrm{CO}\right), 1.96,1.96,1.95,1.93$, $1.91\left(5 \mathrm{~s}, 15 \mathrm{H}, 5 \mathrm{CH}_{3} \mathrm{CO}\right)$. Anal. Calcd for $\mathrm{C}_{79} \mathrm{H}_{79} \mathrm{ClO}_{31}$ : C, 60.83; H, 5.10. Found: C, 60.76; H, 5.04.

\subsection{Acetonyl 4,6-di- $O$-acetyl-2- $O$-benzoyl- $\beta$-D-gluco-} pyranosyl-( $\rightarrow 3$ )-2- $O$-benzoyl-4,6- $O$-benzylidene- $\beta$-Dglucopyranosyl-( $\rightarrow 3)-4,6$-di- $O$-acetyl-2- $O$-benzoyl- $\beta$-Dglucopyranosyl-(1 $\rightarrow 3)$-2- $O$-benzoyl-4,6- $O$-benzylidene- $\alpha$ D-glucopyranoside (23)

Compound 22 (350 $\mathrm{mg}, 0.22 \mathrm{mmol}$ ) was dissolved in mixed solvents of $\mathrm{CH}_{2} \mathrm{Cl}_{2}(4 \mathrm{~mL})$ and $\mathrm{MeOH}(6 \mathrm{~mL})$. To the solution were added thiourea $(85 \mathrm{mg}, 1.04 \mathrm{mmol})$ and 2 ,4-lutidine $(25 \mu \mathrm{L}, 0.23 \mathrm{mmol})$, and the reaction mixture was boiled under reflux for $16 \mathrm{~h}$, at the end of which time TLC (1.5:1 petroleum ether-EtOAc) indicated that the reaction was complete. The mixture was concentrated and extracted with $\mathrm{CH}_{2} \mathrm{Cl}_{2}$, and the organic phase was washed with $\mathrm{N} \mathrm{HCl}$, satd aq $\mathrm{NaHCO}_{3}$, and water, dried, and concentrated. Purification by column chromatography with $2: 1$ petroleum ether-EtOAc as the eluent afforded $23(270 \mathrm{mg}, 81 \%)$ as an amorphous solid: $[\alpha]_{\mathrm{D}}^{20}+20.4\left(c 0.9, \mathrm{CHCl}_{3}\right) ;{ }^{1} \mathrm{H} \mathrm{NMR}: \delta$ 7.79-7.13 $(\mathrm{m}, 30 \mathrm{H}, 4 \mathrm{Bz}-H, 2 \mathrm{Ph}-H), 5.52,5.48(2 \mathrm{~s}, 2 \mathrm{H}, 2 \mathrm{PhCH})$, $5.10-4.86\left(\mathrm{~m}, 5 \mathrm{H}, \mathrm{H}-2^{\mathrm{II}-\mathrm{IV}}, 4^{\mathrm{II}, \mathrm{IV}}\right), 5.05\left(\mathrm{~d}, 1 \mathrm{H}, J_{1,2}\right.$ $\left.3.6 \mathrm{~Hz}, \mathrm{H}-1^{\mathrm{I}}\right), 4.80,4.64,4.62\left(3 \mathrm{~d}, 3 \mathrm{H}, J_{1,2} 7.8 \mathrm{~Hz}, \mathrm{H}-\right.$ $\left.1^{\mathrm{II}-\mathrm{IV}}\right), 4.73\left(\mathrm{dd}, 1 \mathrm{H}, J_{1,2} 3.6 \mathrm{~Hz}, J_{2,3} 9.6 \mathrm{~Hz}, \mathrm{H}-2^{\mathrm{I}}\right)$,
4.35-3.30 (m, 18H, H-3 $\left.{ }^{\mathrm{I}-\mathrm{IV}}, 4^{\mathrm{I}, \mathrm{III}}, 5^{\mathrm{I}-\mathrm{IV}}, 6^{\mathrm{I}-\mathrm{IV}}\right), 4.09$, $4.01\left(\mathrm{ABq}, 2 \mathrm{H}, J 17.3 \mathrm{~Hz}, \mathrm{CH}_{2} \mathrm{COCH}_{3}\right), 2.01,1.96$, 1.96, 1.94, $1.92\left(5 \mathrm{~s}, 15 \mathrm{H}, 5 \mathrm{CH}_{3} \mathrm{CO}\right)$. Anal. Calcd for $\mathrm{C}_{77} \mathrm{H}_{78} \mathrm{O}_{30}$ : C, 62.34; H, 5.30. Found: C, 62.16; H, 5.14.

3.23. Acetonyl $2,3,4,6$-tetra- $O$-benzoyl- $\beta$-D-glucopyranosyl-( $1 \rightarrow 3)-2-O$-benzoyl-4,6- $O$-benzylidene- $\beta$-D-glucopyranosyl-( $1 \rightarrow 3$ )-4,6-di- $O$-acetyl-2- $O$-benzoyl- $\beta$-D-glucopyranosyl-( $1 \rightarrow 3)$-2- $O$-benzoyl-4,6- $O$-benzylidene- $\beta$-D-glucopyranosyl-( $1 \rightarrow 3)-4$,6-di- $O$-acetyl-2- $O$-benzoyl- $\beta$-D-glucopyranosyl-( $\rightarrow 3$ )-2- $O$-benzoyl-4,6- $O$-benzylidene- $\alpha$-Dglucopyranoside (25)

Compound 25 (180 mg, 50\%) was obtained as an amorphous solid from compounds 23 (220 mg, $0.148 \mathrm{mmol})$ and $24(210 \mathrm{mg}, 0.192 \mathrm{mmol})$ according to the general procedure for the coupling reaction. However, purification was carried out with 2:1 petroleum ether-EtOAc as the eluent: $[\alpha]_{\mathrm{D}}^{20}+46.9\left(c 1.0, \mathrm{CHCl}_{3}\right) ;{ }^{1} \mathrm{H} \mathrm{NMR}: \delta 7.92-$ $7.07(\mathrm{~m}, 60 \mathrm{H}, 9 \mathrm{Bz}-H, 3 \mathrm{Ph}-H), 5.50,5.50,5.37(3 \mathrm{~s}, 3 \mathrm{H}$, $3 \mathrm{PhCH}), 5.54\left(\mathrm{dd}, 1 \mathrm{H}, J_{2,3}=J_{3,4}=9.5 \mathrm{~Hz}, \mathrm{H}-3^{\mathrm{VI}}\right), 5.50$ $\left(\mathrm{dd}, 1 \mathrm{H}, J_{3,4}=J_{4,5}=9.5 \mathrm{~Hz}, \mathrm{H}-4^{\mathrm{VI}}\right), 5.33\left(\mathrm{dd}, 1 \mathrm{H}, J_{1,2}\right.$ $\left.8.0 \mathrm{~Hz}, J_{2,3} 9.5 \mathrm{~Hz}, \mathrm{H}-3^{\mathrm{VI}}\right), 5.09-4.62\left(\mathrm{~m}, 7 \mathrm{H}, \mathrm{H}-2^{\mathrm{I}-\mathrm{V}}\right.$, $\left.4^{\mathrm{II}, \mathrm{IV}}\right), 5.04\left(\mathrm{~d}, 1 \mathrm{H}, J_{1,2} 3.6 \mathrm{~Hz}, \mathrm{H}-1^{\mathrm{I}}\right), 4.79,4.75,4.51$, $4.50,4.43\left(5 \mathrm{~d}, 5 \mathrm{H}, J_{1,2} 8.0 \mathrm{~Hz}, \mathrm{H}-1^{\mathrm{II}-\mathrm{VI}}\right), 4.36-3.24(\mathrm{~m}$, $\left.26 \mathrm{H}, \mathrm{H}-3^{\mathrm{I}-\mathrm{V}}, 4^{\mathrm{I}, \mathrm{III}, \mathrm{V}}, 5^{\mathrm{I}-\mathrm{VI}}, 6^{\mathrm{I}-\mathrm{VI}}\right), 4.09,3.97(\mathrm{ABq}, 2 \mathrm{H}$, $\left.J 17.3 \mathrm{~Hz}, \mathrm{CH}_{2} \mathrm{COCH}_{3}\right), 1.96,1.96,1.95,1.89,1.84(5 \mathrm{~s}$, $\left.15 \mathrm{H}, 5 \mathrm{CH}_{3} \mathrm{CO}\right)$. Anal. Calcd for $\mathrm{C}_{131} \mathrm{H}_{122} \mathrm{O}_{45}$ : C, 65.11; H, 5.09. Found: C, 65.26; H, 5.24.

3.24. Acetonyl $2,3,4,6$-tetra- $O$-benzoyl- $\beta$-D-glucopyranosyl-(1 $\rightarrow 3)-2-O$-benzoyl- $\beta$-D-glucopyranosyl- $(1 \rightarrow 3)-4,6-$ di- $O$-acetyl-2- $O$-benzoyl- $\beta$-D-glucopyranosyl- $(1 \rightarrow 3)-2-O$ benzoyl- $\beta$-D-glucopyranosyl-( $\rightarrow 3$ )-4,6-di- $O$-acetyl-2- $O$ benzoyl- $\beta$-D-glucopyranosyl-( $\rightarrow 3)$-2- $O$-benzoyl- $\alpha$-Dglucopyranoside (26)

To a solution of compounds $25(130 \mathrm{mg}, 0.054 \mathrm{mmol})$ and ethylene glycol $(0.1 \mathrm{~mL}, 1.80 \mathrm{mmol})$ in $\mathrm{MeCN}$ $(5 \mathrm{~mL})$ was added $p$-toluenesulfonic acid $\cdot \mathrm{H}_{2} \mathrm{O}(10 \mathrm{mg})$. The mixture was stirred at $\mathrm{rt}$ overnight at the end of which time TLC (1:1 petroleum ether-EtOAc) indicated that the reaction was complete. The mixture was made neutralized with $\mathrm{Et}_{3} \mathrm{~N}$, concentrated, and extracted with $\mathrm{CH}_{2} \mathrm{Cl}_{2}$, the organic phase was washed with water, then dried and concentrated. Purification by column chromatography with $1: 1$ petroleum ether-EtOAc as the eluent afforded compound 26 (80 mg, 69\%) as an amorphous solid: $[\alpha]_{\mathrm{D}}^{20}+16.3\left(c 0.8, \mathrm{CHCl}_{3}\right) ;{ }^{1} \mathrm{H}$ NMR: $\delta$ 8.08-6.97 $(\mathrm{m}, 45 \mathrm{H}, 9 \mathrm{Bz}-H), 5.68\left(\mathrm{dd}, 1 \mathrm{H}, J_{2,3}=J_{3,4}=9.6 \mathrm{~Hz}, \mathrm{H}-\right.$ $\left.3^{\mathrm{VI}}\right), 5.51\left(\mathrm{dd}, 1 \mathrm{H}, J_{3,4}=J_{4,5}=9.6 \mathrm{~Hz}, \mathrm{H}-4^{\mathrm{VI}}\right), 5.38$ $\left(\mathrm{dd}, 1 \mathrm{H}, J_{1,2} 8.0 \mathrm{~Hz}, J_{2,3} 9.6 \mathrm{~Hz}, \mathrm{H}-3^{\mathrm{VI}}\right), 5.06(\mathrm{~d}, 1 \mathrm{H}$, $\left.J_{1,2} 3.8 \mathrm{~Hz}, \mathrm{H}_{-1}{ }^{\mathrm{I}}\right), 5.03-4.62\left(\mathrm{~m}, 7 \mathrm{H}, \mathrm{H}-2^{\mathrm{I}-\mathrm{V}}, 4^{\mathrm{II}, \mathrm{IV}}\right)$, $4.77,4.61,4.59,4.48,4.46\left(5 \mathrm{~d}, 5 \mathrm{H}, J_{1,2} 8.0 \mathrm{~Hz}\right.$, $\left.\mathrm{H}-1^{\mathrm{II}-\mathrm{VI}}\right), 4.35-3.14\left(\mathrm{~m}, 28 \mathrm{H}, \mathrm{CH}_{2} \mathrm{COCH}_{3}, \mathrm{H}-3^{\mathrm{I}-\mathrm{V}}\right.$, $\left.4^{\mathrm{I}, \mathrm{III}, \mathrm{V}}, 5^{\mathrm{I}-\mathrm{VI}}, 6^{\mathrm{I}-\mathrm{VI}}\right), 2.08,2.04,1.88,1.88,1.82(5 \mathrm{~s}$, 
$15 \mathrm{H}, 5 \mathrm{CH} \mathrm{H}_{3} \mathrm{CO}$ ). Anal. Calcd for $\mathrm{C}_{110} \mathrm{H}_{110} \mathrm{O}_{45}: \mathrm{C}, 61.39$; H, 5.15. Found: C, 61.56; H, 5.24.

3.25. Acetonyl 2,3,4,6-tetra- $O$-benzoyl- $\beta$-D-glucopyranosyl-(1 $\rightarrow 3)-[2,3,4,6$-tetra- $O$-benzoyl- $\beta$-D-glucopyranosyl$(1 \rightarrow 6) \mid-2-O$-benzoyl- $\beta$-D-glucopyranosyl- $(1 \rightarrow 3)-4,6-d i-O$ acetyl-2- $O$-benzoyl- $\beta$-D-glucopyranosyl- $(1 \rightarrow 3)-[2,3,4,6$ tetra- $O$-benzoyl- $\beta$-D-glucopyranosyl-( $1 \rightarrow 6) \mid-2-O$-benzoyl$\beta$-D-glucopyranosyl-( $\rightarrow 3)$-4, 6-di- $O$-acetyl-2- $O$-benzoyl$\beta$-D-glucopyranosyl-( $\rightarrow 3)$-[2,3,4,6-tetra- $O$-benzoyl- $\beta$-Dglucopyranosyl-( $(\rightarrow 6) \mid-2-O$-benzoyl- $\alpha$-D-glucopyranoside (27)

Compound 27 (60 mg, 55\%) was obtained as an amorphous solid from compounds $26(60 \mathrm{mg}, 0.0279 \mathrm{mmol})$ and $9(75 \mathrm{mg}, 0.1 \mathrm{mmol})$ according to the general procedure for the coupling reaction. However, purification was carried out with 1:1.5 petroleum ether-EtOAc as the eluent: $[\alpha]_{\mathrm{D}}^{20}+46.9\left(c\right.$ 1.2, $\left.\mathrm{CHCl}_{3}\right) ;{ }^{1} \mathrm{H}$ NMR: $\delta$ 8.05-6.87 (m, 105H, 21Bz-H), 5.95-5.29 (m, 12H, H$\left.2^{\mathrm{VI}-\mathrm{IX}}, 3^{\mathrm{VI}-\mathrm{IX}}, 4^{\mathrm{VI}-\mathrm{IX}}\right), 4.97-4.37\left(\mathrm{~m}, 15 \mathrm{H}, \mathrm{H}-1^{\mathrm{II}-\mathrm{IX}}, 2^{\mathrm{I}-\mathrm{V}}\right.$, $\left.4^{\mathrm{IIIV}}\right), 4.78\left(\mathrm{~d}, 1 \mathrm{H}, J_{1,2} 3.6 \mathrm{~Hz}, \mathrm{H}-1^{\mathrm{I}}\right), 4.26-3.10(\mathrm{~m}$, $\left.37 \mathrm{H}, \mathrm{CH}_{2} \mathrm{COCH}_{3}, \mathrm{H}-3^{\mathrm{I}-\mathrm{V}}, 4^{\mathrm{I}, \mathrm{III}, \mathrm{V}}, 5^{\mathrm{I}-\mathrm{IX}}, 6^{\mathrm{I}-\mathrm{IX}}\right), 2.03$, 2.03, 1.99, 1.86, $1.81\left(5 \mathrm{~s}, 15 \mathrm{H}, 5 \mathrm{CH}_{3} \mathrm{CO}\right) .{ }^{13} \mathrm{C}$ NMR: $\delta$ $170.5,170.4,170.4,169.3,169.2\left(5 \mathrm{C}, 5 \mathrm{CH}_{3} \mathrm{CO}\right), 166.1$, $166.0,165.9,165.7,165.7,165.7,165.6,165.5,165.2$, $165.2,165.1,165.1,165.0,164.9,164.9,164.8,164.6$, $163.8,163.5,163.3,163.1(21 \mathrm{~s}, 21 \mathrm{C}, \mathrm{BzCO}), 102.1$, $102.1,101.5,101.4,101.4,100.9,100.7,100.5$ (8C, $\beta-C-$ 1), 95.3 (1C, $\alpha-C-1), 22.65,21.62,20.63,20.45,20.41$ (5C, $5 \mathrm{CH}_{3} \mathrm{CO}$ ). Anal. Calcd for $\mathrm{C}_{212} \mathrm{H}_{188} \mathrm{O}_{72}: \mathrm{C}, 65.49$; $\mathrm{H}, 4.87$. Found: $\mathrm{C}, 65.66 ; \mathrm{H}, 4.74$.

3.26. Acetonyl $\beta$-D-glucopyranosyl-( $1 \rightarrow 3)-[\beta$-D-glucopyranosyl-( $1 \rightarrow 6)]-\beta$-D-glucopyranosyl-( $(1 \rightarrow 3)-\beta$-D-glucopyranosyl-( $1 \rightarrow 3)$-[ $\beta$-D-glucopyranosyl-( $1 \rightarrow 6)]-\beta$-D-glucopyranosyl-( $1 \rightarrow 3)$ - $\beta$-D-glucopyranosyl-( $1 \rightarrow 3)$-[ $\beta$-D-glucopyranosyl-( $(1 \rightarrow 6)]-\alpha$-D-glucopyranoside $(28)$

Satd $\mathrm{NH}_{3}$ in $\mathrm{MeOH}(5 \mathrm{~mL})$ was added to compounds 27 $(40 \mathrm{mg}, 10.29 \mu \mathrm{mol})$ in $\mathrm{MeOH}(4 \mathrm{~mL})$. After $48 \mathrm{~h}$ at $\mathrm{rt}$, the reaction mixture was concentrated, and the residue was purified by Sephadex LH-20 chromatography
$(\mathrm{MeOH})$ to afford $\mathbf{2 8}$ as an amorphous solid $(15.5 \mathrm{mg}$, 98\%): $[\alpha]_{\mathrm{D}}^{20}+2.9\left(c 1.0, \mathrm{H}_{2} \mathrm{O}\right) ;{ }^{1} \mathrm{H}$ NMR $\left(\mathrm{D}_{2} \mathrm{O}\right): \delta 4.95$ $\left(\mathrm{d}, 1 \mathrm{H}, J_{1,2} 3.9 \mathrm{~Hz}, \mathrm{H}-1^{\mathrm{I}}\right), 4.77,4.77,4.76,4.76,4.53$, $4.53,4.49,4.49\left(8 \mathrm{~d}, 8 \mathrm{H}, J_{1,2} 8.0 \mathrm{~Hz}, \mathrm{H}-1^{\mathrm{II}-\mathrm{IX}}\right), 4.07(\mathrm{~s}$, $\left.2 \mathrm{H}, \mathrm{CH}_{3} \mathrm{COCH}_{2}\right), 4.23-3.30\left(\mathrm{~m}, 54 \mathrm{H}, \mathrm{H}-2^{\mathrm{I}-\mathrm{IX}}, 3^{\mathrm{I}-\mathrm{IX}}\right.$, $\left.4^{\mathrm{I}-\mathrm{IX}}, 5^{\mathrm{I}-\mathrm{IX}}, 6^{\mathrm{I}-\mathrm{IX}}\right), 1.99\left(\mathrm{~s}, 3 \mathrm{H}, \mathrm{CH}_{3} \mathrm{CO}\right) .{ }^{13} \mathrm{C} \mathrm{NMR}$ $\left(\mathrm{D}_{2} \mathrm{O}\right): \delta 172.10\left(1 \mathrm{C}, \mathrm{CH}_{3} \mathrm{COCH}_{2}\right), 102.6,102.6,102.6$, $102.5,102.3,102.3,102.2,102.2(8 \mathrm{C}, \beta-\mathrm{C}-1), 97.98$ (1C, $\alpha-C-1), 84.57,84.50,84.00,83.80,81.55$ (5C, C$\left.3^{\mathrm{A}-\mathrm{E}}\right), 21.03$ (s, $\left.1 \mathrm{C}, \mathrm{CH}_{3} \mathrm{COCH}_{2}\right)$. Anal. Calcd for $\mathrm{C}_{57} \mathrm{H}_{96} \mathrm{O}_{47}$ : C, 44.65; H, 6.31. Found: $\mathrm{C}, 44.76 ; \mathrm{H}, 6.44$.

\section{Acknowledgements}

This work was supported by The Chinese Academy of Sciences (KZCX3-J-08) and by The National Natural Science Foundation of China (Projects 30070185 and 39970864).

\section{References}

1. Sasaki, T.; Takasuka, N. Carbohydr. Res. 1976, 47, 99104.

2. (a) Norisue, T.; Yanaki, T.; Fujita, H. J. Polym. Sci. 1980, 18, 547-552; (b) Kojima, T.; Tabata, K.; Itoh, W.; Yanaki, T. Agric. Biol. Chem. 1986, 50, 231-238; (c) Brown, G. D.; Gordon, S. Nature 2001, 413, 36-37; (d) Willment, J. A.; Gordon, S.; Brown, G. D. J. Biol. Chem. 2001, 276, 4381843823; (e) Mueller, A.; Raptis, J.; Rice, P. J.; Kalbfleisch, J. H.; Stout, R. D.; Ensley, H. E.; Browder, W.; Williams, D. L. Glycobiology 2000, 10, 339-346.

3. Saito, H.; Ohki, T.; Takasuka, N.; Sasaki, T. A. Carbohydr. Res. 1977, 58, 293-305.

4. Yang, G.; Kong, F. Synlett 2000, 1423-1426.

5. Ning, J.; Zhang, W.; Yi, Y.; Yang, G.; Wu, Z.; Yi, J.; Kong, F. Bioorg. Med. Chem. 2003, 11, 2193-2203.

6. Zhao, W.; Kong, F. Carbohydr. Res. 2003, 338, 28132823.

7. Schmidt, R. R.; Kinzy, W. Adv. Carbohydr. Chem. Biochem. 1994, 50, 121-125.

8. Hosomi, A.; Kobayashi, H.; Sakurai, H. Tetrahedron Lett. 1980, 955-958.

9. (a) Zeng, Y.; Ning, J.; Kong, F. Tetrahedron Lett. 2002 , 3729-3733; (b) Zeng, Y.; Ning, J.; Kong, F. Carbohydr. Res. 2003, 338, 307-311. 